\title{
A NOTION OF COMPATIBILITY FOR ARMENDARIZ AND BAER PROPERTIES OVER SKEW PBW EXTENSIONS
}

\author{
ARMANDO REYES AND HÉCTOR SUÁREZ
}

\begin{abstract}
In this paper we are interested in studying the properties of Armendariz, Baer, quasi-Baer, p.p. and p.q.-Baer over skew PBW extensions. Using a notion of compatibility, we generalize several propositions established for Ore extensions and present new results for several noncommutative rings which can not be expressed as Ore extensions (universal enveloping algebras, diffusion algebras, and others).
\end{abstract}

\section{INTRODUCTION}

In 22, Kaplansky defined a ring $B$ as a Baer (resp. quasi-Baer, which was defined by Clark [10]) ring, if the right annihilator of every nonempty subset (resp. ideal) of $B$ is generated by an idempotent (the objective of these rings is to abstract various properties of von Neumann algebras and complete $*$-regular rings; Clark used the quasi-Baer concept to characterize when a finite-dimensional algebra with unity over an algebraically closed field is isomorphic to a twisted matrix units semigroup algebra). Another generalization of Baer rings are the p.p.-rings. A ring $B$ is called right (resp. left) p.p., if the right (resp. left) annihilator of each element of $B$ is generated by an idempotent (or equivalently, rings in which each principal right (resp. left) ideal is projective). Birkenmeier et al. [8] defined a ring to be called a right (resp. left) principally quasi-Baer (or simply right (resp. left) p.q.-Baer) ring, if the right annihilator of each principal right (resp. left) ideal of $B$ is generated by an idempotent. Note that in a reduced ring $B, B$ is Baer (resp. p.p.) if and only if $B$ is quasi-Baer (resp. p.q.-Baer); see [5] for more details.

Commutative and noncommutative Baer, quasi-Baer, p.p. and p.q.-Baer rings have been investigated in the literature. For instance, in [3], Armendariz established the following proposition: if $B$ is a reduced ring, then $B[x]$ is a Baer ring if and only if $B$ is a Baer ring ([3] Theorem B]; Armendariz showed an example to illustrate that the condition to be reduced is not superfluous). Birkenmeier et al. in [8] showed that the quasi-Baer condition is preserved by many polynomial extensions, and

2010 Mathematics Subject Classification. Primary: 16S36; 16E50. Secondary: 16W60.

Key words and phrases. Armendariz, Baer, quasi-Baer, p.p. and p.q.-Baer rings, skew PBW extensions.

The first named author was supported by the research fund of Departamento de Matemáticas, Universidad Nacional de Colombia, Bogotá, Colombia, HERMES code 30366. 
in [6], they proved that a ring $B$ is right p.q.-Baer if and only if $B[x]$ is right p.q.Baer. In the context of noncommutative rings, more exactly polynomial extensions known as Ore extensions $B[x ; \sigma, \delta]$ of injective type, i.e., when $\sigma$ is injective, we found several works (cf. [10, 5, 6, 7, 13, 19, 8, 9, 17, 18, 15, 16]). Some of these works consider the case $\delta=0$ and $\sigma$ an automorphism, or the case where $\sigma$ is the identity. It is important to say that the Baerness and quasi-Baerness of a ring $B$ and an Ore extension $B[x ; \sigma, \delta]$ of $B$ do not depend on each other. More exactly, there are examples which show that there exists a Baer ring $B$ but the Ore extension $B[x ; \sigma, \delta]$ is not right p.q.-Baer; similarly, there exist Ore extensions $B[x ; \sigma, \delta]$ which are quasi-Baer, but $B$ is not quasi-Baer (see [19] for more details).

One of the most important kinds of rings for which all the above properties have been studied are the $\sigma$-rigid rings (cf. [24, 19, 18]). Following Krempa [24, an endomorphism $\sigma$ of a ring $B$ is called rigid if $a \sigma(a)=0$ implies $a=0$, for $a \in B$, and a ring $B$ is said to be $\sigma$-rigid, if there exists a rigid endomorphism $\sigma$ of $B$. One can see that any rigid endomorphism of a ring is a monomorphism, and $\sigma$-rigid rings are reduced rings ([19, p. 218]). With the aim of generalizing the $\sigma$-rigid rings in the context of Ore extensions, in [2] Annin introduced the notion of compatibility: a ring $B$ is called $\sigma$-compatible if for every $a, b \in B$, we have $a b=0$ if and only if $a \sigma(b)=0$ (necessarily, the endomorphism $\sigma$ is injective); $B$ is called $\delta$-compatible if for each $a, b \in B, a b=0 \Rightarrow a \delta(b)=0$. If $B$ is both $\sigma$-compatible and $\delta$-compatible, $B$ is called $(\sigma, \delta)$-compatible. Note that $\sigma$-rigid rings are $(\sigma, \delta)$ compatible rings ([15, Lemma 3.3]), but the converse is false ([15. Examples 2.1, 2.2 and 2.3]). Nevertheless, Hashemi et al., in [16, Lemma 2.2], showed that a ring $B$ is $(\sigma, \delta)$-compatible and reduced if and only if $B$ is $\sigma$-rigid. Hence $\sigma$-compatible rings generalize $\sigma$-rigid rings for the case where $B$ is not assumed to be reduced. All the above properties have been also studied for $(\sigma, \delta)$-compatible rings: in [16] it was imposed the $(\sigma, \delta)$-compatibility on the $\operatorname{ring} B$ and it was proved that (i) the ring $B$ is quasi-Baer if and only if $B[x ; \sigma, \delta]$ is quasi-Baer; (ii) the ring $B$ is left p.q.-Baer if and only if $B[x ; \sigma, \delta]$ is left p.q.-Baer. In this way, the treatment in [16] is a generalization of [6, Theorem 1.8] and [9, Theorem 3.1].

With all the above facts in mind, a natural question for a given class of Baer, quasi-Baer, p.p. and p.q.-Baer rings is their behavior with respect to skew PoincaréBirkhoff-Witt (PBW for short) extensions introduced by Gallego and Lezama in [12, which are more general than Ore extensions of injective type. More exactly, it has been shown that skew PBW extensions contain various well-known groups of algebras such as some types of Auslander-Gorenstein rings, some skew Calabi-Yau algebras, some Artin-Schelter regular algebras, some Koszul algebras, quantum polynomials, some quantum universal enveloping algebras, etc. (see [27] and [34 for a detailed list of examples). In fact, these extensions include several algebras which cannot be expressed as Ore extensions: universal enveloping algebras of finite Lie algebras, diffusion algebras, and others; see Section 5 This fact shows the necessity to have more general results in a theory of Baerness and quasi-Baerness for several noncommutative rings. As a matter of fact, several ring 
and module theoretical properties have been studied for skew PBW extensions (cf. [29]-45]).

Precisely, a first treatment about these topics was established by the first author in [35]. There, a notion of rigidness ( $\Sigma$-rigid rings) was the key concept to establish necessary and sufficient conditions to guarantee that all these properties are stable over skew PBW extensions. In this way, the results presented in [35] generalize the treatment presented in 19 about $\sigma$-rigid rings. A second treatment is developed by the authors in 38 , with the aim of generalizing $\Sigma$-rigid rings (compare also with [29]). There, the important notions are skew Armendariz rings and a more general class of rings, the weak skew Armendariz rings ([38], Definitions 3.1 and 3.2, respectively). Using these two notions, the authors studied the properties of being Baer, quasi-Baer, p.p. and p.q.-Baer over skew PBW extensions and over their classical ring of quotients.

Now, as we saw above, $(\sigma, \delta)$-compatible rings generalize $\sigma$-rigid rings for the case of Ore extensions, and since we are interested in a more general family of noncommutative rings, the skew PBW extensions, a natural problem is to establish a notion of compatibility for these extensions. With this aim, we introduce the $(\Sigma, \Delta)$-compatible rings which are a natural generalization of $(\sigma, \delta)$-compatible rings, since skew PBW extensions generalize Ore extensions of injective type. Of course, $(\Sigma, \Delta)$-compatible rings generalize $\Sigma$-rigid rings (Proposition 3.4). It is important to say that the treatment developed in [38] is not a particular case of the treatment developed in this paper, and vice versa, the results obtained in this paper cannot be obtained from the treatment in [38. In other words, there are examples of skew Armendariz rings which are not $(\Sigma, \Delta)$-compatible rings, and there are examples of $(\Sigma, \Delta)$-compatible rings which are not skew Armendariz rings (see Remark 3.7 for more details). Therefore, this paper can be considered as a second approach to Armendariz skew PBW extensions.

Let us briefly explain the content of this second approach to a notion of Armendariz ring for skew PBW extensions. Let us start with the commutative definition. In commutative algebra, a ring $B$ is called Armendariz (the term was introduced by Rege and Chhawchharia in 30) if whenever polynomials $f(x)=$ $a_{0}+a_{1} x+\cdots+a_{n} x^{n}, g(x)=b_{0}+b_{1} x+\cdots+b_{m} x^{m} \in B[x]$ satisfy $f(x) g(x)=0$, then $a_{i} b_{j}=0$, for every $i, j$. The interest of this notion lies in its natural and useful role in understanding the relation between the annihilators of the ring $B$ and the annihilators of the polynomial ring $B[x]$. In [3, Lemma 1], Armendariz showed that a reduced ring (i.e., a ring without nonzero nilpotent elements) always satisfies this condition (reduced rings are Abelian, that is, every idempotent is central, and also semiprime, that is, its prime radical is trivial). Now, in the context of noncommutative algebra, more exactly the Ore extensions, the relations between Armendariz rings and Baer (quasi-Baer) rings have been also investigated in different papers, see for example [3, 4, 30, 1, 6, 13, 19, 23, 8, 18, 17, 20, 28, . For instance, Hirano in [18] defined a ring $B$ to be quasi-Armendariz if whenever two polynomials $f(x)=\sum_{i=0}^{m} a_{i} x^{i}, g(x)=\sum_{j=0}^{t} b_{j} x^{j} \in B[x]$ satisfy $f(x) B[x] g(x)=0$, then $a_{i} R b_{j}$, for every $i, j$. 
Motivated by all these results, Hashemi et al. investigated in [15] a notion of Armendariz ring as a generalization of the $\sigma$-rigid rings. There, it was introduced the condition (SA1) which is a skew polynomial version of Armendariz rings. More exactly: if $\sigma$ is a monomorphism of a ring $B$ and $\delta$ is a $\sigma$-derivation of $B$, then it is said that $B$ satisfies the (SA1) condition if whenever $f(x) g(x)=0$ for $f(x)=$ $\sum_{i=0}^{m} a_{i} x^{i}$ and $g(x)=\sum_{j=0}^{n} b_{j} x^{j} \in B[x ; \sigma, \delta]$, then $a_{i} b_{j}=0$, for all $i, j$. Since $\sigma$-rigid rings satisfy (SA1) ([19, Proposition 6]), in [15] it was imposed the $(\sigma, \delta)$ compatibility on the ring $B$, and it was shown the following facts: (i) if $B$ satisfies (SA1), then $B$ is a Baer (resp. right p.p.) ring if and only if $B[x ; \sigma, \delta]$ is a Baer (resp. right p.p.) ring ([15, Theorem 3.14]). In this way, this result is a generalization of [19, Theorems 11, 14, and Corollaries 12, 16]; (ii) if (SA1) holds in B, the ring $B$ satisfies the ascending chain condition on right annihilators if and only if so does $B[x ; \sigma, \delta]$ ([15, Theorem 2.5]). Hashemi et al. in [16] investigated also a generalization of $\sigma$-rigid rings by introducing the condition (SQA1) which is a skew polynomial version of the quasi-Armendariz rings. More exactly, for a monomorphism $\sigma$ and a $\sigma$-derivation $\delta$ of a ring $B$, it is said that $B$ satisfies the (SQA1) condition if whenever $f(x) B[x ; \sigma, \delta] g(x)=0$ for $f(x)=\sum_{i=0}^{m} a_{i} x^{i}$ and $g(x)=\sum_{j=0}^{t} b_{j} x^{j} \in B[x ; \sigma, \delta]$, then $a_{i} B b_{j}=0$, for all $i, j$. Note that if $B$ is $\sigma$-rigid, then $B$ satisfies also (SQA1). With all these works in mind, our second approach to a notion of Armendariz ring of skew PBW extensions consists in establishing the conditions (SA1) and (SQA1) for the case of skew PBW extensions with the aim of generalizing the results presented in [35] about $\Sigma$-rigid rings, and of course, the results presented in [15] and [16] for Ore extensions of injective type.

Next, we describe the structure of the article. In Section 2 we establish some useful results about skew PBW extensions for the rest of the paper. In Section 3 . we introduce the notion of $\Sigma$-compatible, $\Delta$-compatible, and $(\Sigma, \Delta)$-compatible ring (Definition 3.2), and we show that $\Sigma$-rigid rings defined in [35] are strictly contained in $(\Sigma, \Delta)$-compatible rings (Proposition 3.4 and Example 3.6. However, in Theorem 3.9 we prove the following equivalences: for a skew PBW extension $A$ of a ring $R, R$ is $(\Sigma, \Delta)$-compatible and reduced $\Leftrightarrow R$ is $\Sigma$-rigid $\Leftrightarrow A$ is reduced. In Section 4, we introduce the notions (SA1) and (SQA1) for skew PBW extensions (Definitions 4.1 and 4.11. respectively) with the aim of generalizing the results presented in [35] about Baer, quasi-Baer, p.p. and p.q.-Baer rings for $\Sigma$-rigid rings, to the more general setting of $(\Sigma, \Delta)$-compatible rings, see Theorems $4.2,4.4,4.7$ and 4.15 In Example 4.10 we show an example of a $\sigma$-compatible left p.q.-Baer ring which is not $\sigma$-rigid. We also study some relations between the condition (SQA1) and annihilators and ideals in a ring $R$ and a skew PBW extension $A$ over $R$ (Theorems 4.12 and 4.14). Finally, in Section 5 we present some examples of noncommutative rings which cannot be expressed as Ore extensions but are skew PBW extensions. As a matter of fact, the results presented in this paper are new for skew PBW extensions and all of them are similar to others existing in the literature for the case of Ore extensions. In this way, we continue the task of studying homological and ring properties of skew PBW extensions (cf. $[12,32,27,33,34,26,35,36,38$ ). 


\section{SKEW PBW EXTENSIONS}

Definition 2.1 ([12, Definition 1]). Let $R$ and $A$ be rings. We say that $A$ is a skew $P B W$ extension of $R$ (also called a $\sigma$-PBW extension of $R$ ), which is denoted by $A:=\sigma(R)\left\langle x_{1}, \ldots, x_{n}\right\rangle$, if the following conditions hold:

(i) $R \subseteq A$;

(ii) there exist elements $x_{1}, \ldots, x_{n} \in A$ such that $A$ is a left free $R$-module, with basis $\operatorname{Mon}(A):=\left\{x^{\alpha}=x_{1}^{\alpha_{1}} \cdots x_{n}^{\alpha_{n}} \mid \alpha=\left(\alpha_{1}, \ldots, \alpha_{n}\right) \in \mathbb{N}^{n}\right\}$, and $x_{1}^{0} \cdots x_{n}^{0}:=1 \in \operatorname{Mon}(A)$.

(iii) For each $1 \leq i \leq n$ and any $r \in R \backslash\{0\}$, there exists an element $c_{i, r} \in$ $R \backslash\{0\}$ such that $x_{i} r-c_{i, r} x_{i} \in R$.

(iv) For any positive integers $1 \leq i, j \leq n$, there exists $c_{i, j} \in R \backslash\{0\}$ such that $x_{j} x_{i}-c_{i, j} x_{i} x_{j} \in R+R x_{1}+\cdots+R x_{n}$.

Proposition 2.2 ([12, Proposition 3]). Let $A$ be a skew PBW extension of $R$. For each $1 \leq i \leq n$, there exist an injective endomorphism $\sigma_{i}: R \rightarrow R$ and a $\sigma_{i}$-derivation $\delta_{i}: R \rightarrow R$ such that $x_{i} r=\sigma_{i}(r) x_{i}+\delta_{i}(r)$, for each $r \in R$. We write $\Sigma:=\left\{\sigma_{1}, \ldots, \sigma_{n}\right\}$ and $\Delta:=\left\{\delta_{1}, \ldots, \delta_{n}\right\}$.

Definition 2.3 ([12, Definition 4]). Let $A$ be a skew PBW extension of $R$.

(a) $A$ is called quasi-commutative if the conditions (iii) and (iv) in Definition 2.1 are replaced by the following conditions: (iii') for each $1 \leq i \leq n$ and all $r \in R \backslash\{0\}$, there exists $c_{i, r} \in R \backslash\{0\}$ such that $x_{i} r=c_{i, r} x_{i}$; (iv') for any $1 \leq i, j \leq n$, there exists $c_{i, j} \in R \backslash\{0\}$ such that $x_{j} x_{i}=c_{i, j} x_{i} x_{j}$.

(b) $A$ is called bijective, if $\sigma_{i}$ is bijective for each $1 \leq i \leq n$ and $c_{i, j}$ is invertible, for any $1 \leq i<j \leq n$.

Example 2.4. The class of skew PBW extensions contains various well-known groups of algebras such as some types of Auslander-Gorenstein rings, some skew Calabi-Yau algebras, Koszul algebras, quantum polynomials, some quantum universal enveloping algebras, etc. A detailed list of examples of skew PBW extensions is presented in [27] and in [34].

Definition 2.5 ([12, Definition 6]). Let $A$ be a skew PBW extension of $R$ with endomorphisms $\sigma_{i}, 1 \leq i \leq n$, as in Proposition 2.2

(i) For $\alpha=\left(\alpha_{1}, \ldots, \alpha_{n}\right) \in \mathbb{N}^{n}, \sigma^{\alpha}:=\sigma_{1}^{\alpha_{1}} \cdots \sigma_{n}^{\alpha_{n}},|\alpha|:=\alpha_{1}+\cdots+\alpha_{n}$. If $\beta=\left(\beta_{1}, \ldots, \beta_{n}\right) \in \mathbb{N}^{n}$, then $\alpha+\beta:=\left(\alpha_{1}+\beta_{1}, \ldots, \alpha_{n}+\beta_{n}\right)$.

(ii) For $X=x^{\alpha} \in \operatorname{Mon}(A), \exp (X):=\alpha, \operatorname{deg}(X):=|\alpha|$, and $X_{0}:=1$. The symbol $\succeq$ will denote a total order defined on $\operatorname{Mon}(A)$ (a total order on $\left.\mathbb{N}^{n}\right)$. For an element $x^{\alpha} \in \operatorname{Mon}(A), \exp \left(x^{\alpha}\right):=\alpha \in \mathbb{N}^{n}$. If $x^{\alpha} \succeq x^{\beta}$ but $x^{\alpha} \neq x^{\beta}$, we write $x^{\alpha} \succ x^{\beta}$. Every element $f \in A$ can be expressed uniquely as $f=a_{0}+a_{1} X_{1}+\cdots+a_{m} X_{m}$, with $a_{i} \in R$, and $X_{m} \succ \cdots \succ X_{1}$. With this notation, we define $\operatorname{lm}(f):=X_{m}$, the leading monomial of $f$; $\operatorname{lc}(f):=a_{m}$, the leading coefficient of $f ; \operatorname{lt}(f):=a_{m} X_{m}$, the leading term of $f ; \exp (f):=\exp \left(X_{m}\right)$, the order of $f$; and $E(f):=\left\{\exp \left(X_{i}\right) \mid 1 \leq i \leq t\right\}$. Note that $\operatorname{deg}(f):=\max \left\{\operatorname{deg}\left(X_{i}\right)\right\}_{i=1}^{t}$. Finally, if $f=0$, then $\operatorname{lm}(0):=0$, $\operatorname{lc}(0):=0, \operatorname{lt}(0):=0$. We also consider $X \succ 0$ for any $X \in \operatorname{Mon}(A)$. For a 
detailed description of monomial orders in skew PBW extensions, see [12, Section 3].

Proposition 2.6 ([12, Theorem 7]). If $A$ is a polynomial ring with coefficients in $R$ and the set of indeterminates $\left\{x_{1}, \ldots, x_{n}\right\}$, then $A$ is a skew PBW extension of $R$ if and only if the following conditions hold:

(i) for each $x^{\alpha} \in \operatorname{Mon}(A)$ and every $0 \neq r \in R$, there exist unique elements $r_{\alpha}:=\sigma^{\alpha}(r) \in R \backslash\{0\}, p_{\alpha, r} \in A$, such that $x^{\alpha} r=r_{\alpha} x^{\alpha}+p_{\alpha, r}$, where $p_{\alpha, r}=0$, or $\operatorname{deg}\left(p_{\alpha, r}\right)<|\alpha|$ if $p_{\alpha, r} \neq 0$. If $r$ is left invertible, so is $r_{\alpha}$.

(ii) For each $x^{\alpha}, x^{\beta} \in \operatorname{Mon}(A)$, there exist unique elements $c_{\alpha, \beta} \in R$ and $p_{\alpha, \beta} \in A$ such that $x^{\alpha} x^{\beta}=c_{\alpha, \beta} x^{\alpha+\beta}+p_{\alpha, \beta}$, where $c_{\alpha, \beta}$ is left invertible, $p_{\alpha, \beta}=0$, or $\operatorname{deg}\left(p_{\alpha, \beta}\right)<|\alpha+\beta|$ if $p_{\alpha, \beta} \neq 0$.

Remark 2.7. With respect to Proposition 2.6, we have two observations:

(i) ([35, Proposition 2.9]) If $\alpha:=\left(\alpha_{1}, \ldots, \alpha_{n}\right) \in \mathbb{N}^{n}$ and $r \in R$, then

$$
\begin{aligned}
x^{\alpha} r= & x_{1}^{\alpha_{1}} x_{2}^{\alpha_{2}} \cdots x_{n-1}^{\alpha_{n-1}} x_{n}^{\alpha_{n}} r=x_{1}^{\alpha_{1}} \cdots x_{n-1}^{\alpha_{n-1}}\left(\sum_{j=1}^{\alpha_{n}} x_{n}^{\alpha_{n}-j} \delta_{n}\left(\sigma_{n}^{j-1}(r)\right) x_{n}^{j-1}\right) \\
& +x_{1}^{\alpha_{1}} \cdots x_{n-2}^{\alpha_{n-2}}\left(\sum_{j=1}^{\alpha_{n-1}} x_{n-1}^{\alpha_{n-1}-j} \delta_{n-1}\left(\sigma_{n-1}^{j-1}\left(\sigma_{n}^{\alpha_{n}}(r)\right)\right) x_{n-1}^{j-1}\right) x_{n}^{\alpha_{n}} \\
& +x_{1}^{\alpha_{1}} \cdots x_{n-3}^{\alpha_{n-3}}\left(\sum_{j=1}^{\alpha_{n-2}} x_{n-2}^{\alpha_{n-2}-j} \delta_{n-2}\left(\sigma_{n-2}^{j-1}\left(\sigma_{n-1}^{\alpha_{n-1}}\left(\sigma_{n}^{\alpha_{n}}(r)\right)\right)\right) x_{n-2}^{j-1}\right) x_{n-1}^{\alpha_{n-1}} x_{n}^{\alpha_{n}} \\
& +\cdots+x_{1}^{\alpha_{1}}\left(\sum_{j=1}^{\alpha_{2}} x_{2}^{\alpha_{2}-j} \delta_{2}\left(\sigma_{2}^{j-1}\left(\sigma_{3}^{\alpha_{3}}\left(\sigma_{4}^{\alpha_{4}}\left(\cdots\left(\sigma_{n}^{\alpha_{n}}(r)\right)\right)\right)\right) x_{2}^{j-1}\right) x_{3}^{\alpha_{3}} x_{4}^{\alpha_{4}} \cdots x_{n-1}^{\alpha_{n-1}} x_{n}^{\alpha_{n}}\right. \\
& +\sigma_{1}^{\alpha_{1}}\left(\sigma_{2}^{\alpha_{2}}\left(\cdots\left(\sigma_{n}^{\alpha_{n}}(r)\right)\right)\right) x_{1}^{\alpha_{1}} \cdots x_{n}^{\alpha_{n}}, \quad \sigma_{j}^{0}:=\mathrm{id}_{R} \quad \text { for } 1 \leq j \leq n .
\end{aligned}
$$

(ii) ([35, Remark 2.10]) Using (i), it follows that for the product $a_{i} X_{i} b_{j} Y_{j}$, if $X_{i}:=x_{1}^{\alpha_{i 1}} \cdots x_{n}^{\alpha_{i n}}$ and $Y_{j}:=x_{1}^{\beta_{j 1}} \cdots x_{n}^{\beta_{j n}}$, then

$$
\begin{aligned}
a_{i} X_{i} b_{j} Y_{j}= & a_{i} \sigma^{\alpha_{i}}\left(b_{j}\right) x^{\alpha_{i}} x^{\beta_{j}}+a_{i} p_{\alpha_{i 1}, \sigma_{i 2}^{\alpha_{i 2}}\left(\cdots\left(\sigma_{i n}^{\alpha_{i n}}(b)\right)\right)} x_{2}^{\alpha_{i 2}} \cdots x_{n}^{\alpha_{i n}} x^{\beta_{j}} \\
& +a_{i} x_{1}^{\alpha_{i 1}} p_{\alpha_{i 2}, \sigma_{3}^{\alpha_{i 3}}\left(\cdots \left(\sigma_{i n}^{\left.\left.\alpha_{i n}(b)\right)\right)}\right.\right.} x_{3}^{\alpha_{i 3}} \cdots x_{n}^{\alpha_{i n}} x^{\beta_{j}} \\
& +a_{i} x_{1}^{\alpha_{i 1}} x_{2}^{\alpha_{i 2}} p_{\alpha_{i 3}, \sigma_{i 4}^{\alpha_{i 4}}\left(\cdots\left(\sigma_{i n}^{\alpha_{i n}}(b)\right)\right)} x_{4}^{\alpha_{i 4}} \cdots x_{n}^{\alpha_{i n}} x^{\beta_{j}} \\
& +\cdots+a_{i} x_{1}^{\alpha_{i 1}} x_{2}^{\alpha_{i 2}} \cdots x_{i(n-2)}^{\alpha_{i(n-2)}} p_{\alpha_{i(n-1)}, \sigma_{i n}^{\alpha_{i n}}(b)} x_{n}^{\alpha_{i n}} x^{\beta_{j}} \\
& +a_{i} x_{1}^{\alpha_{i 1}} \cdots x_{i(n-1)}^{\alpha_{i(n-1)}} p_{\alpha_{i n}, b} x^{\beta_{j}}
\end{aligned}
$$

In this way, when we compute every summand of $a_{i} X_{i} b_{j} Y_{j}$ we obtain products of the coefficient $a_{i}$ with several evaluations of $b_{j}$ in $\sigma$ 's and $\delta$ 's depending on the coordinates of $\alpha_{i}$.

\section{3. $(\Sigma, \Delta)$-COMPATIBLE RINGS}

Following Krempa [24, an endomorphism $\sigma$ of a ring $B$ is called rigid if $a \sigma(a)=0$ implies $a=0$, for $a \in B$. A ring $B$ is said to be $\sigma$-rigid if there exists a rigid 
endomorphism $\sigma$ of $B$. It is clear that any rigid endomorphism of a ring is a monomorphism, and $\sigma$-rigid rings are reduced ([19, p. 218]). Properties of $\sigma$-rigid rings have been studied by several authors (cf. [24, 19, 18]). With this in mind, in [2] it is said that $B$ is $\sigma$-compatible if for every $a, b \in B$, we have $a b=0$ if and only if $a \sigma(b)=0 ; B$ is said to be $\delta$-compatible if for each $a, b \in B, a b=0 \Rightarrow a \delta(b)=0$. If $B$ is both $\sigma$-compatible and $\delta$-compatible, $B$ is called $(\sigma, \delta)$-compatible. In this case, the endomorphism $\sigma$ is injective. Since one can appreciate the relation between these notions and $\sigma$-rigid rings ([15, Lemma 3.3]), in [16, Lemma 2.2] it was shown that a ring $B$ is $(\sigma, \delta)$-compatible and reduced if and only if $B$ is $\sigma$-rigid. Hence $\sigma$-compatible rings generalize $\sigma$-rigid rings for the case where $B$ is not assumed to be reduced. The natural task for us is to extend this notion of compatibility to a more general context of Ore extensions of injective type, that is, the family of skew PBW extensions. This is precisely the content of Definition 3.2 Before, we recall the notion of $\Sigma$-rigid ring.

Definition 3.1 ([35, Definition 3.2]). Let $B$ be a ring and $\Sigma$ a family of endomorphisms of $B . \Sigma$ is called a rigid endomorphisms family if $r \sigma^{\alpha}(r)=0$ implies $r=0$, for every $r \in B$ and $\alpha \in \mathbb{N}^{n}$. A ring $B$ is called $\Sigma$-rigid if there exists a rigid endomorphisms family $\Sigma$ of $B$.

Note that if $\Sigma$ is a rigid endomorphisms family, then every element $\sigma_{i} \in \Sigma$ is a monomorphism. In fact, $\Sigma$-rigid rings are reduced rings: if $B$ is a $\Sigma$-rigid ring and $r^{2}=0$ for $r \in B$, then $0=r \sigma^{\alpha}\left(r^{2}\right) \sigma^{\alpha}\left(\sigma^{\alpha}(r)\right)=r \sigma^{\alpha}(r) \sigma^{\alpha}(r) \sigma^{\alpha}\left(\sigma^{\alpha}(r)\right)=$ $r \sigma^{\alpha}(r) \sigma^{\alpha}\left(r \sigma^{\alpha}(r)\right)$, i.e., $r \sigma^{\alpha}(r)=0$ and so $r=0$, that is, $B$ is reduced (note that there exists an endomorphism of a reduced ring which is not a rigid endomorphism, see [19, Example 9]). We consider the family of injective endomorphisms $\Sigma$ and the family $\Delta$ of $\Sigma$-derivations in a skew PBW extension $A$ of a ring $R$ (see Proposition 2.2.

Definition 3.2. Consider a ring $R$ with a family of endomorphisms $\Sigma$ and a family of $\Sigma$-derivations $\Delta$. Then,

(1) $R$ is said to be $\Sigma$-compatible, if for each $a, b \in R, a \sigma^{\alpha}(b)=0$ if and only if $a b=0$, for every $\alpha \in \mathbb{N}^{n}$;

(2) $R$ is said to be $\Delta$-compatible, if for each $a, b \in R, a b=0$ implies $a \delta^{\beta}(b)=0$, for every $\beta \in \mathbb{N}^{n}$.

If $R$ is both $\Sigma$-compatible and $\Delta$-compatible, $R$ is called $(\Sigma, \Delta)$-compatible.

Examples 3.3. Next, we present remarkable examples of $\sigma$-PBW extensions over $(\Sigma, \Delta)$-compatible rings (see 31] or [27] for a detailed definition and reference of every example).

(a) If $A$ is a skew PBW extension of a ring $R$ where the coefficients commute with the variables, that is, $x_{i} r=r x_{i}$, for every $r \in R$ and each $i=1, \ldots, n$, or equivalently, $\sigma_{i}=\operatorname{id}_{R}$ and $\delta_{i}=0$, for every $i$ (these extensions were called constant by the authors in [44), then it is clear that $R$ is $(\Sigma, \Delta)$-compatible. Some examples of constant $\sigma$-PBW extensions are the following: PBW extensions defined by Bell and Goodearl 
(which include the classical commutative polynomial rings, universal enveloping algebra of a Lie algebra, and others); some operator algebras (for example, the algebra of linear partial differential operators, the algebra of linear partial shift operators, the algebra of linear partial difference operators, the algebra of linear partial $q$-dilation operators, and the algebra of linear partial $q$-differential operators); the class of diffusion algebras; Weyl algebras; additive analogue of the Weyl algebra; multiplicative analogue of the Weyl algebra; some quantum Weyl algebras as $A_{2}\left(J_{a, b}\right)$; the quantum algebra $\mathcal{U}^{\prime}(\mathfrak{s o}(3, \mathbb{k}))$; the family of 3 -dimensional skew polynomial algebras (there are exactly fifteen of these algebras, see [39]); Dispin algebra $\mathcal{U}(\operatorname{osp}(1,2))$; Woronowicz algebra $\mathcal{W}_{v}(\mathfrak{s l}(2, \mathbb{k}))$; the complex algebra $V_{q}\left(\mathfrak{s l}_{3}(\mathbb{C})\right) ; q$-Heisenberg algebra $\mathbf{H}_{n}(q)$; the Hayashi algebra $W_{q}(J)$, and more.

(b) We also encounter examples of $\sigma$-PBW extensions (which are not constant) over $(\Sigma, \Delta)$-compatible rings. Let us see: (i) the quantum plane $\mathcal{O}_{q}\left(\mathbb{k}^{2}\right)$; the algebra of $q$-differential operators $D_{q, h}[x, y]$; the mixed algebra $D_{h}$; the operator differential rings; the algebra of differential operators $D_{\mathbf{q}}\left(S_{\mathbf{q}}\right)$ on a quantum space $S_{\mathbf{q}}$, and more.

(c) It is important to say that several algebras of quantum physics can be expressed as skew PBW extensions (for instance, Weyl algebras, additive and multiplicative analogue of the Weyl algebra, quantum Weyl algebras, $q$-Heisenberg algebra, and others), which allows us to characterize several properties with physical meaning. As Curado et al. say in [11, "algebraic methods have long been applied to the solution of a large number of quantum physical systems. In the last decades, quantum algebras appeared in the framework of quantum integrable one-dimensional models and have ever since been applied to many physical phenomena [...] It was found that it could be generalized leading to the concept of deformed Heisenberg algebras that have been used in many areas, as nuclear physics, condensed matter, atomic physics, etc." With these ideas in mind, next we present some remarkable examples of these algebras which are $(\Sigma, \Delta)$-compatible (the proof that these algebras are skew PBW extensions can be realized using the theory developed in [40]).

(i) The Lie-deformed Heisenberg algebra introduced by Jannussis is defined by the commutation relations

$$
\begin{aligned}
q_{j}\left(1+i \lambda_{j k}\right) p_{k}-p_{k}\left(1-i \lambda_{j k}\right) q_{j} & =i \hbar \delta_{j k} \\
{\left[q_{j}, q_{k}\right] } & =\left[p_{j}, p_{k}\right]=0, \quad j, k=1,2,3,
\end{aligned}
$$

where $q_{j}, p_{j}$ are the position and momentum operators, and $\lambda_{j k}=$ $\lambda_{k} \delta_{j k}$, with $\lambda_{k}$ real parameters. If $\lambda_{j k}=0$ one recovers the usual Heisenberg algebra.

(ii) The quantum Weyl algebra introduced by Giaquinto and Zhang with the aim of studying the Jordan Hecke symmetry is a quantization of the usual second Weyl algebra. By definition, $A_{2}\left(J_{a, b}\right)$ is the $\mathbb{k}$-algebra generated by the variables $x_{1}, x_{2}, \partial_{1}, \partial_{2}$, with relations (depending on 


$$
\begin{array}{rlrl}
\quad & \text { parameters } a, b \in \mathbb{k}) & & \\
x_{1} x_{2} & =x_{2} x_{1}+a x_{1}^{2}, & & \partial_{2} \partial_{1}=\partial_{1} \partial_{2}+b \partial_{2}^{2} \\
\partial_{1} x_{1}=1+x_{1} \partial_{1}+a x_{1} \partial_{2}, & & \partial_{1} x_{2}=-a x_{1} \partial_{1}-a b x_{1} \partial_{2}+x_{2} \partial_{1}+b x_{2} \partial_{2} \\
\partial_{2} x_{1} & =x_{1} \partial_{2}, & & \partial_{2} x_{2}=1-b x_{1} \partial_{2}+x_{2} \partial_{2} .
\end{array}
$$

Over any field $\mathbb{k}$, if $a=b=0$, then $A_{2}\left(J_{0,0}\right) \cong A_{2}$, the usual second Weyl algebra.

(iii) With the purpose of obtaining bosonic representations of the DrinfeldJimbo quantum algebras, Hayashi considered the algebra U. Let us see its construction. Let $\mathbf{U}$ be the algebra generated by the indeterminates $\omega_{1}, \ldots, \omega_{n}, \psi_{1}, \ldots, \psi_{n}, \psi_{1}^{*}, \ldots, \psi_{n}^{*}$, with the relations

$$
\begin{array}{rlrl}
\psi_{j} \psi_{i}-\psi_{i} \psi_{j} & =\psi_{j}^{*} \psi_{i}^{*}-\psi_{i}^{*} \psi_{j}^{*}=\omega_{j} \omega_{i}-\omega_{i} \omega_{j}=\psi_{j}^{*} \psi_{i}-\psi_{i} \psi_{j}^{*}=0, & 1 \leq i<j \leq n, \\
\omega_{j} \psi_{i}-q^{-\delta_{i j}} \psi_{i} \omega_{j}=\psi_{j}^{*} \omega_{i}-q^{-\delta_{i j}} \omega_{i} \psi_{j}^{*}=0, & 1 \leq i, j \leq n, \\
\psi_{i}^{*} \psi_{i}-q^{2} \psi_{i} \psi_{i}^{*}=-q^{2} \omega_{i}^{2}, \quad q \in \mathbb{C} & 1 \leq i \leq n .
\end{array}
$$

(iv) Jannussis et al. studied the non-Hermitian realization of a Lie deformed, non-canonical Heisenberg algebra, considering the case of operators $A_{j}, B_{k}$ which are non-Hermitian (i.e., $\hbar=1$ )

$$
\begin{aligned}
A_{j}\left(1+i \lambda_{j k}\right) B_{k}-B_{k}\left(1-i \lambda_{j k}\right) A_{j} & =i \delta_{j k} \\
{\left[A_{j}, B_{k}\right] } & =0 \quad(j \neq k) \\
{\left[A_{j}, A_{k}\right] } & =\left[B_{j}, B_{k}\right]=0,
\end{aligned}
$$

and

$$
\begin{aligned}
A_{j}^{+}\left(1+i \lambda_{j k}\right) B_{k}^{+}-B_{k}^{+}\left(1-i \lambda_{j k}\right) A_{j}^{+} & =i \delta_{j k} \\
{\left[A_{j}^{+}, B_{k}^{+}\right] } & =0 \quad(j \neq k), \\
{\left[A_{j}^{+}, A_{k}^{+}\right] } & =\left[B_{j}^{+}, B_{k}^{+}\right]=0,
\end{aligned}
$$

where $A_{j} \neq A_{j}^{+}, B_{k} \neq B_{k}^{+}(j, k=1,2,3)$. If the operators $A_{j}, B_{k}$ are in the form $A_{j}=f_{j}\left(N_{j}+1\right) a_{j}, B_{k}=a_{k}^{+} f_{k}\left(N_{k}+1\right)$, where $a_{j}, a_{j}^{+}$are leader operators of the usual Heisenberg-Weyl algebra, with $N_{j}$ the corresponding number operator $\left(N_{j}=a_{j}^{+} a_{j}, N_{j}\left|n_{j}\right\rangle=n_{j}\left|n_{j}\right\rangle\right)$, and the structure functions $f_{j}\left(N_{j}+1\right)$ complex, then it is showed that $A_{j}$ and $B_{k}$ are given by

$$
\begin{aligned}
A_{j} & =\sqrt{\frac{i}{1+i \lambda_{j}}}\left(\frac{\left[\left(1-i \lambda_{j}\right) /\left(1+i \lambda_{j}\right)\right]^{N_{j}+1}-1}{\left(1-i \lambda_{j}\right) /\left(1+i \lambda_{j}\right)-1} \frac{1}{N_{j}+1}\right)^{\frac{1}{2}} a_{j} \\
B_{k} & =\sqrt{\frac{i}{1+i \lambda_{k}}} a_{k}^{+}\left(\frac{\left[\left(1-i \lambda_{k}\right) /\left(1+i \lambda_{k}\right)\right]^{N_{k}+1}-1}{\left(1-i \lambda_{k}\right) /\left(1+i \lambda_{k}\right)-1} \frac{1}{N_{k}+1}\right)^{\frac{1}{2}} .
\end{aligned}
$$

Proposition 3.4 shows that $(\Sigma, \Delta)$-compatible rings are a generalization of $\Sigma$ rigid rings introduced in [35, Definition 3.2].

Proposition 3.4. Let $\Sigma$ be a family of endomorphisms of a ring $R$, and let $\Delta$ be a family of $\Sigma$-derivations of $R$. If $R$ is $\Sigma$-rigid, then $R$ is $(\Sigma, \Delta)$-compatible. 
Proof. If $R$ is a $\Sigma$-rigid ring, then $R$ is reduced, and $a b=0$ if and only if $b a=0$. In this way, $a \sigma^{\alpha}(b) \sigma^{\alpha}\left(a \sigma^{\alpha}(b)\right)=a \sigma^{\alpha}(b a) \sigma^{\alpha}\left(\sigma^{\alpha}(b)\right)=0$, whence $a \sigma^{\alpha}(b)=0$. Using a similar reasoning, we can see that the equality $b a=0$ implies $\sigma^{\alpha}(a) b=0$ : $0=\sigma^{\alpha}\left(\sigma^{\alpha}(a)\right) \sigma^{\alpha}(b a) b=\sigma^{\alpha}\left(\sigma^{\alpha}(a)\right) \sigma^{\alpha}(b) \sigma^{\alpha}(a) b=\sigma^{\alpha}\left(\sigma^{\alpha}(a) b\right) \sigma^{\alpha}(a) b \Rightarrow \sigma^{\alpha}(a) b=$ 0 . Now, if $a \sigma^{\alpha}(b)=0$, then $b a \sigma^{\alpha}(b) \sigma^{\alpha}(a)=b a \sigma^{\alpha}(b a)=0$, whence $b a=0$, and then $a b=0$. Finally, for every $i, 0=\delta_{i}(b a)=\delta_{i}(b) a+\sigma_{i}(b) \delta_{i}(a)$, that is, $\left(\sigma_{i}(b) \delta_{i}(a)\right)^{2}=-\delta_{i}(b) a \sigma_{i}(b) \delta_{i}(a)=0$, and since $R$ is reduced, $\sigma_{i}(b) \delta_{i}(a)=0$, i.e., $\delta_{i}(b) a=0$, which shows that $a \delta_{i}(b)=0$.

Corollary 3.5 ([15, Lemma 3.3]). Let $\sigma$ be an endomorphism of a ring $B$ and let $\delta$ be a $\sigma$-derivation of $B$. If $B$ is $\sigma$-rigid, then $B$ is $(\sigma, \delta)$-compatible.

The following example illustrates that the converse of Proposition 3.4 is false. See also Examples 4.17 (iii).

Example 3.6 ([15, Example 2.2]). Let $\delta$ be a $\sigma$-derivation of $B$, where $B$ is a $\sigma$-rigid ring. Consider

$$
B_{3}=\left\{\left(\begin{array}{lll}
a & b & c \\
0 & a & d \\
0 & 0 & a
\end{array}\right) \mid a, b, c, d \in B\right\},
$$

the subring of the upper triangular matrix $T_{3}(B)$. The endomorphism $\sigma$ of $B$ is extended to the endomorphism $\bar{\sigma}: B_{3} \rightarrow B_{3}$ defined by $\bar{\sigma}\left(\left(a_{i j}\right)\right)=\left(\sigma\left(a_{i j}\right)\right)$ and the $\sigma$-derivation $\delta$ of $B$ is also extended to $\bar{\delta}: B_{3} \rightarrow B_{3}$ defined by $\bar{\delta}\left(\left(a_{i j}\right)\right)=\left(\delta\left(a_{i j}\right)\right)$. Then $\bar{\delta}$ is a $\bar{\sigma}$-derivation of $B_{3}$, and we have the following facts: (i) $B_{3}$ is a $(\bar{\sigma}, \bar{\delta})$ compatible ring; (ii) $B_{3}$ is not $\bar{\sigma}$-rigid (in fact, $B_{3}$ satisfies (SA1), see Definition 4.1 ).

Remark 3.7. As we said in the Introduction, the treatment developed in [38, is not a particular case of the treatment developed in this paper, and vice versa, the results obtained in this paper cannot be obtained from the treatment in [38. In other words, there are examples of skew Armendariz rings which are not $(\Sigma, \Delta)$ compatible rings, and there are examples of $(\Sigma, \Delta)$-compatible rings which are not skew Armendariz rings. For example:

(a) [46, Example 2.8] If $\mathbb{Z}_{2}[x]$ is the polynomial ring with coefficients in $\mathbb{Z}_{2}$, and $\sigma$ is the endomorphism of $\mathbb{Z}_{2}[x]$ given by $\sigma(p(x))=p(0)$, then $\mathbb{Z}_{2}[x]$ is skew Armendariz [38, Definition 3.1] but $\mathbb{Z}_{2}[x]$ is not $\Sigma=\{\sigma\}$-compatible (so not $\Sigma$-rigid): if we take $f=\overline{1}+x, g=x \in \mathbb{Z}_{2}[x]$, then $f g=(\overline{1}+x) x \neq 0$, but $f \sigma(g)=(\overline{1}+x) \sigma(x)=0$.

(b) [46, Example 2.9] Let $\mathbb{Z}_{4}$ be the ring of integers modulo 4 . Consider the ring

$$
R=\left\{\left(\begin{array}{ll}
a & \bar{b} \\
0 & a
\end{array}\right) \mid a \in \mathbb{Z}, \bar{b} \in \mathbb{Z}_{4}\right\} .
$$

If we consider the endomorphism $\sigma$ of $R$ given by $\sigma\left(\left(\begin{array}{cc}a & \bar{b} \\ 0 & a\end{array}\right)\right)=\left(\begin{array}{cc}a & -b \\ 0 & a\end{array}\right)$, and the ideal $I=\left\{\left(\begin{array}{ll}a & \overline{0} \\ 0 & a\end{array}\right) \mid a \in 4 \mathbb{Z}\right\}$ of $R$, then $R / I$ is not skew Armendariz, and $R / I$ is $\Sigma=\{\bar{\sigma}\}$-compatible. 
Returning to the relation between $\Sigma$-rigid and $(\Sigma, \Delta)$-compatible rings, from Proposition 3.4 and Example 3.6, note that

$$
\Sigma \text {-rigid rings } \varsubsetneqq(\Sigma, \Delta) \text {-compatible rings. }
$$

Next, we investigate some key properties of $(\Sigma, \Delta)$-compatible rings.

Proposition 3.8. Let $R$ be $a(\Sigma, \Delta)$-compatible ring. For every $a, b \in R$, we have:

(i) if $a b=0$, then $a \sigma^{\theta}(b)=\sigma^{\theta}(a) b=0$, for each $\theta \in \mathbb{N}^{n}$.

(ii) If $\sigma^{\beta}(a) b=0$ for some $\beta \in \mathbb{N}^{n}$, then $a b=0$.

(iii) If $a b=0$, then $\sigma^{\theta}(a) \delta^{\beta}(b)=\delta^{\beta}(a) \sigma^{\theta}(b)=0$, for every $\theta, \beta \in \mathbb{N}^{n}$.

Proof. The proof uses a similar argument to the one established in [15, Lemma 2.4].

As we saw before, $\Sigma$-rigid rings are strictly contained in $(\Sigma, \Delta)$-compatible rings. Nevertheless, Theorem 3.9 shows the importance of reduced rings in the equivalence of both families of rings.

Theorem 3.9. If $A$ is a skew $P B W$ extension of a ring $R$, then the following statements are equivalent:

(i) $R$ is reduced and $(\Sigma, \Delta)$-compatible;

(ii) $R$ is $\Sigma$-rigid;

(iii) $A$ is reduced.

Proof. (i) $\Rightarrow$ (ii) Suppose that $R$ is reduced and $(\Sigma, \Delta)$-compatible. Consider an element $r \in R$ such that $r \sigma^{\alpha}(r)=0$. From Proposition 3.8 (i) we obtain $\sigma^{\alpha}(r) \sigma^{\alpha}(r)$ for every $\alpha \in \mathbb{N}$, and using the injectivity of $\sigma^{\alpha}$ and the assumption on $R$, we have $r=0$. (ii) $\Rightarrow$ (i) It follows from Proposition 3.4 (ii) $\Rightarrow$ (iii) Let $R$ be $\Sigma$ rigid and suppose that $A$ is not reduced. Then there exists a non-zero element $f \in A$ such that $f^{2}=0$. Since $R$ is reduced, $f \notin R$. Following Definition 2.5. consider $f=a_{0}+a_{1} X_{1}+\cdots+a_{m} X_{m}, a_{i} \in R, 0 \leq i \leq m, a_{m} \neq 0$, with $X_{i}=x^{\alpha_{i}}=x_{1}^{\alpha_{i 1}} \cdots x_{n}^{\alpha_{i n}}$, and $X_{m} \succ X_{m-1} \succ \cdots \succ X_{1}$. By Theorem 2.6 (ii) we have

$$
\begin{aligned}
f^{2} & =\left(a_{m} X_{m}+\cdots+a_{1} X_{1}+a_{0}\right)\left(a_{m} X_{m}+\cdots+a_{1} X_{1}+a_{0}\right) \\
& =a_{m} X_{m} a_{m} X_{m}+\text { other terms of order less than } X_{m} X_{m} \\
& =a_{m}\left[\sigma^{\alpha_{m}}\left(a_{m}\right) X_{m}+p_{\alpha_{m}, a_{m}}\right] X_{m}+\cdots \\
& =a_{m} \sigma^{\alpha_{m}}\left(a_{m}\right) X_{m} X_{m}+a_{m} p_{\alpha_{m}, a_{m}} X_{m}+\cdots \\
& =a_{m} \sigma^{\alpha_{m}}\left(a_{m}\right)\left[c_{\alpha_{m}, \alpha_{m}} x^{2 \alpha_{m}}+p_{\alpha_{m}, \alpha_{m}}\right]+a_{m} p_{\alpha_{m}, a_{m}} X_{m}+\cdots,
\end{aligned}
$$

where $p_{\alpha_{m}, a_{m}}=0$ or $\operatorname{deg}\left(p_{\alpha_{m}, a_{m}}\right)<\left|\alpha_{m}\right|$ if $p_{\alpha_{m}, a_{m}} \neq 0$, and $p_{\alpha_{m}, \alpha_{m}}=0$ or $\operatorname{deg}\left(p_{\alpha_{m}, \alpha_{m}}\right)<\left|\alpha_{m}+\alpha_{m}\right|$ if $p_{\alpha_{m}, \alpha_{m}} \neq 0$. From the equality

$$
\operatorname{lc}\left(f^{2}\right)=a_{m} \sigma^{\alpha_{m}}\left(a_{m}\right) c_{\alpha_{m}, \alpha_{m}}=0
$$

we obtain $a_{m} \sigma^{\alpha_{m}}\left(a_{m}\right)=0$ ( $A$ is bijective). From [35, Lemma 3.3 (iv)], we obtain $a_{m}^{2}=0$, and so $a_{m}=0$ ( $R$ is reduced), which is a contradiction. Hence, $A$ is reduced. (iii) $\Rightarrow$ (ii) If $A$ is reduced, $R$ is also reduced as a subring. Let us see that 
$R$ is $\Sigma$-rigid. If $a \in R$ and $a \sigma^{\alpha}(a)=0$, then $0=\sigma^{\alpha}(a) x^{\alpha} a \sigma^{\alpha}(a) x^{\alpha} a=\left(\sigma^{\alpha}(a) x^{\alpha} a\right)^{2}$, and so $\sigma^{\alpha}(a) x^{\alpha} a=0$. Thus, $0=\sigma^{\alpha}(a) x^{\alpha} a=\sigma^{\alpha}(a)\left[\sigma^{\alpha}(a) x^{\alpha}+p_{\alpha, a}\right]=\left(\sigma^{\alpha}(a)\right)^{2} x^{\alpha}+$ $\sigma^{\alpha}(a) p_{\alpha, a}$, with $p_{\alpha, a}=0$, or $\operatorname{deg}\left(p_{\alpha, a}\right)<|\alpha|$ if $p_{\alpha, a} \neq 0$ (Theorem 2.6). Hence $\left(\sigma^{\alpha}(a)\right)^{2}=0$, that is, $\sigma^{\alpha}(a)=0$. Now, since $\sigma^{\alpha}$ is injective, we obtain $a=0$, which shows that $R$ is $\Sigma$-rigid.

Corollary 3.10 ([16, Lemma 2.2]). If $\sigma$ is an endomorphism and $\delta$ is a $\sigma$ derivation of a ring $B$, then $B$ is $(\sigma, \delta)$-compatible and reduced if and only if $B$ is $\sigma$-rigid.

Lemma 3.11. Let $A$ be a skew $P B W$ extension of a $(\Sigma, \Delta)$-compatible ring $R$. If $f=a_{0}+a_{1} X_{1}+\cdots+a_{m} X_{m} \in A, r \in R$, and $f r=0$, then $a_{i} r=0$, for every $i$.

Proof. Consider the expression $f r$ with $X_{m} \succ \cdots \succ X_{1}$. Then

$$
\begin{aligned}
f r & =\left(a_{0}+a_{1} X_{1}+\cdots+a_{m} X_{m}\right) r=a_{0} r+a_{1} X_{1} r+\cdots+a_{m} X_{m} r \\
& =a_{0} r+a_{1}\left(\sigma^{\alpha_{1}}(r) X_{1}+p_{\alpha_{1}, r}\right)+\cdots+a_{m}\left(\sigma^{\alpha_{m}}(r) X_{m}+p_{\alpha_{m}, r}\right) \\
& =a_{0} r+a_{1} \sigma^{\alpha_{1}}(r) X_{1}+a_{1} p_{\alpha_{1}, r}+\cdots+a_{m} \sigma^{\alpha_{m}}(r) X_{m}+a_{m} p_{\alpha_{m}, r},
\end{aligned}
$$

where $p_{\alpha_{j}, r}=0$, or $\operatorname{deg}\left(p_{\alpha_{j}, r}\right)<|\alpha|$ if $p_{\alpha_{j}, r} \neq 0$, for $j=1, \ldots, m$ (Proposition 2.6). Note that $\operatorname{lc}(f r)=a_{m} \sigma^{\alpha_{m}}(r)=0$, and by the $\Sigma$-compatibility of $R, a_{m} r=0$, that is, $r$ is an element of the right annihilator of $a_{m}$. From Remark 2.7, the polynomial $p_{\alpha_{m}, r}$ involves elements obtained evaluating $\sigma$ 's and $\delta$ 's (depending on the coordinates of $\left.\alpha_{m}\right)$ in the element $r$. Hence, by $(\Sigma, \Delta)$-compatibility of $R$ and Proposition 3.8, we obtain $a_{m} p_{\alpha_{m}, r}=0$, which means that the expression $f r$ takes the form $f r=a_{0} r+a_{1} X_{1} r+\cdots+a_{m-1} X_{m-1} r$. Using this argument repeatedly, we can see that $a_{i} r=0$, for every $i=0, \ldots, m$.

Corollary 3.12 ([16, Lemma 2.3]). Let $B$ be a $(\sigma, \delta)$-compatible ring. If $f(x)=$ $a_{0}+a_{1} x+\cdots+a_{m} x^{m} \in B[x ; \sigma, \delta], r \in B$ and $f(x) r=0$, then $a_{i} r=0$, for each $i$.

\section{BAER, QUASI-BAER, P.P. AND P.Q.-RINGS}

In [35, the first author studied skew PBW extensions of Baer, quasi-Baer, p.p. and p.q.-Baer rings over $\Sigma$-rigid rings. There, it was proved that these properties are stable over this kind of extensions. Now, since we have showed that $\Sigma$-rigid rings are strictly contained in $(\Sigma, \Delta)$-compatible rings, the purpose of this section is to generalize the results presented in [35] to the more general setting of $(\Sigma, \Delta)$-compatible rings. In this way, we obtain more general results for skew PBW extensions and extend the results presented in [9] and [15] for Ore extensions of injective type.

Definition 4.1. Let $A$ be a skew PBW extension of $R$. We say that $R$ satisfies the condition (SA1) if whenever $f g=0$ for $f=a_{0}+a_{1} X_{1}+\cdots+a_{m} X_{m}$ and $g=b_{0}+b_{1} Y_{1}+\cdots+b_{t} Y_{t}$ elements of $A$, then $a_{i} b_{j}=0$, for every $i, j$.

Note that every $\Sigma$-rigid ring satisfies the condition (SA1).

Theorem 4.2. If $A$ is a skew $P B W$ extension of a $(\Sigma, \Delta)$-compatible ring $R$ which satisfies (SA1), then $R$ is a Baer ring if and only if $A$ is a Baer ring. 
Proof. Suppose that $R$ is a Baer ring. Consider a nonempty subset $D$ of $A$, and let $C$ be the set of coefficients of elements of $D$. By assumption on $R$, there exists an idempotent $e \in R$ with right annihilator of $C$ in $R$ given by $r_{R}(C)=e R$. Since $R$ is $(\Sigma, \Delta)$-compatible, from Remark 2.7 (ii) and Proposition 3.8, we can see that $D e=0$, that is, $e A \subseteq r_{A}(D)$. Now, if $f=a_{0}+a_{1} X_{1}+\cdots+a_{m} X_{m} \in r_{A}(D)$, by condition (SA1) on $R, C a_{i}=0$ for $0 \leq i \leq m$, i.e., $a_{i} \in e R$, whence $r_{A}(D) \subseteq e A$, and then $r_{A}(D)=e A$.

Suppose that $A$ is a Baer ring and let $C$ be a nonempty subset of $R$. There exists an idempotent $e \in A$ given by $e=e_{0}+e_{1} X_{1}+\cdots+e_{t} X_{t}$ with $r_{A}(C)=e A$. Hence $C e_{0}=0$, which implies that $e_{0}=e e_{0}$, and by the condition (SA1) on $R$, $e_{0}^{2}=e_{0}$, i.e., $e_{0} R \subseteq r_{R}(C)$. Now, if $r^{\prime} \in r_{R}(C)$, then $r^{\prime}=e r^{\prime}$, that is, $e_{0} r^{\prime}=r^{\prime}$, whence $r_{R}(C)=e_{0} R$.

Corollary 4.3 ([15, Theorem 3.14]). If $B$ is a $(\sigma, \delta)$-compatible ring which satisfies the condition (SA1), then $B$ is a Baer ring if and only if $B[x ; \sigma, \delta]$ is a Baer ring.

Theorem 4.4. If $A$ is a skew $P B W$ extension of a $(\Sigma, \Delta)$-compatible ring $R$ which satisfies the condition (SA1), then $R$ satisfies the ascending chain condition on right annihilators if and only if so does $A$.

Proof. Consider a chain of right annihilators of $A$ given by $I_{1} \subseteq I_{2} \subseteq \cdots$. There exist non-empty subsets $D_{i}$ of $A$ with $r_{A}\left(D_{i}\right)=I_{i}$, for $i \geq 1$. Note that $\cdots \subseteq D_{2} \subseteq$ $D_{1}$. Let $C_{i}$ be the set of coefficients of elements of $D_{i}$, for every $i$. By assumption on $R$, there exists $n \in \mathbb{N}$ such that $r_{R}\left(C_{n}\right)=r_{R}\left(C_{i}\right)$, for $i \geq n$. The aim is to show that $r_{A}\left(D_{n}\right)=r_{A}\left(D_{i}\right)$, for $i \geq n$. With this in mind, let $i \geq n$, and consider the element $g \in r_{A}\left(D_{i}\right)$ given by $g=r_{0}+r_{1} X_{1}+\cdots+r_{m} X_{m}$. Then $h g=0$, for every $h \in D_{i}$. If $h=b_{0}+b_{1} Y_{1}+\cdots+b_{t} Y_{t}$, using the condition (SA1) on $R, 0=b_{k} r_{j}$ $(0 \leq k \leq t, 0 \leq j \leq m)$, that is, $C_{i} r_{j}=0$, for $0 \leq j \leq m$, which shows that $r_{j} \in r_{R}\left(C_{n}\right)=r_{R}\left(C_{i}\right)$, for $0 \leq j \leq m$. Therefore, by the $(\Sigma, \Delta)$-compatibility of $R$ and Proposition 3.8, we have $g \in r_{A}\left(D_{n}\right)$, i.e., $r_{A}\left(D_{n}\right)=r_{A}\left(D_{i}\right)$.

Now, if $J_{1} \subseteq J_{2} \subseteq \cdots$ is a chain of right annihilators of $R$, then there exist nonempty subsets of $R$ with $r_{R}\left(B_{i}\right)=J_{i}$, for $i \geq 1$, and $\cdots \subseteq B_{2} \subseteq B_{1}$. By assumption on $A, r_{A}\left(B_{i}\right)=r_{A}\left(B_{n}\right)$, for some $n \in \mathbb{N}$, and every value $i \geq n$. This fact and the equalities $r_{R}\left(B_{i}\right)=R \cap r_{R}\left(B_{i}\right)=R \cap r_{R}\left(B_{n}\right)=r_{R}\left(B_{n}\right)$, for $i \geq n$, allow us to guarantee that $J_{i}=J_{n}$, for $i \geq n$.

Corollary 4.5 ([15, Theorem 2.5]). If $B$ is a $(\sigma, \delta)$-compatible ring which satisfies the condition (SA1), then $B$ satisfies the ascending chain condition on right annihilators if and only if so does $B[x ; \sigma, \delta]$.

Since a $\Sigma$-rigid ring is a $(\Sigma, \Delta)$-compatible ring and satisfies (SA1), we obtain the following corollary.

Corollary 4.6 ([35, Theorem 2.9]). If $A$ is a bijective skew PBW extension of a $\Sigma$-rigid ring $R$, then $R$ is a Baer ring if and only if $A$ is a Baer ring.

Theorem 4.7. If $A$ is a skew $P B W$ extension of a $(\Sigma, \Delta)$-compatible ring $R$ which satisfies (SA1), then $R$ is a right p.p.-ring if and only if $A$ is a right p.p.-ring. 
Proof. Suppose that $A$ is a right p.p.-ring, and consider an element $a$ of $R$. There exists an idempotent $e=e_{0}+e_{1} X_{1}+\cdots+e_{m} X_{m} \in A$ such that $r_{A}(\{a\})=e A$. The idea is to show that $r_{R}(\{a\})=e_{0} R$. Since $a e=0$, in particular, $a e_{0}=0$, which shows that $e_{0} R \subseteq r_{R}(\{a\})$. Now, consider $r^{\prime} \in r_{R}(\{a\})$. Then $r^{\prime}=e r^{\prime}$, and by the condition (SA1) on $R, r^{\prime}=e_{0} r^{\prime}$. Hence $r_{R}(\{a\})=e_{0} R$, that is, $R$ is Baer.

Suppose that $R$ is a right p.p.-ring and consider an element $f \in A$ given by the expression $f=a_{0}+a_{1} X_{1}+\cdots+a_{t} X_{t}$. Let us see that $R$ is an Abelian ring. Consider $e^{2}=e, r \in R$. Then $(e r(1-e))^{2}=(e r-e r e)(e r-e r e)=$ erer-erere-ereer + ereere $=$ erer-erere - erer + erere $=0$. Using the $(\Sigma, \Delta)$ compatibility of $R$, the equality $e(e-1)=0$ implies $e \sigma_{i}(e)=e$, and $e \delta_{i}(e)=0$, and from the equality $(\operatorname{er}(1-e))^{2}=0$ we obtain $\operatorname{er}(1-e) \sigma_{i}(\operatorname{er}(1-e))=0$ and $(\operatorname{er}(1-e)) \delta_{i}(\operatorname{er}(1-e))=0$ for every $1 \leq i \leq n$. Let $f=e\left(1-e r(1-e) x_{i}\right)$ and $g=\left(1+e r(1-e) x_{i}\right)(1-e)$. Then

$$
\begin{aligned}
f g & =e\left(1-e r(1-e) x_{i}\right)\left(1+e r(1-e) x_{i}\right)(1-e) \\
& =e\left(1+e r(1-e) x_{i}-e r(1-e) x_{i}-e r(1-e) x_{i} \operatorname{er}(1-e) x_{i}\right)(1-e) \\
& =e\left(1-e r(1-e)\left[\sigma_{i}(\operatorname{er}(1-e)) x_{i}+\delta_{i}(\operatorname{er}(1-e)) x_{i}\right]\right)(1-e) \\
& =e\left(1-e r(1-e) \sigma_{i}(\operatorname{er}(1-e)) x_{i}^{2}-e r(1-e) \delta_{i}(\operatorname{er}(1-e))\right) x_{i}(1-e) \\
& =e(1-e)=0 .
\end{aligned}
$$

Note that

$$
\begin{aligned}
f & =e\left(1-e r(1-e) x_{i}\right)=e-e^{2} r(1-e) x_{i}=e+(e r e-e r) x_{i} \\
g & =\left(1+e r(1-e) x_{i}\right)(1-e)=1-e+e r(1-e) x_{i}(1-e) \\
& =1-e+e r(1-e)\left[\sigma_{i}(1-e) x_{i}+\delta_{i}(1-e)\right] \\
& =1-e+(e r-e r e)\left(1-\sigma_{i}(e)\right) x_{i}+(e r-e r e)\left(-\delta_{i}(e)\right) \\
& =1-e+\left(e r e \delta_{i}(e)-e r \delta_{i}(e)\right)+\left(e r-e r \sigma_{i}(e)-e r e+e r e \sigma_{i}(e)\right) x_{i} \\
& =1-e-e r \delta_{i}(e)+\left(e r-e r \sigma_{i}(e)\right) x_{i} .
\end{aligned}
$$

By assumption, $R$ satisfies the condition (SA1), so $e\left(e r-e r \sigma_{i}(e)\right)=0$, that is, $e r-\operatorname{er} \sigma_{i}(e)=e r\left(1-\sigma_{i}(e)\right)=e r \sigma_{i}(1-e)=0$, and hence $\sigma_{i}(e r) \sigma_{i}(1-e)=0$ (Proposition 3.8 (i)), which shows that $\operatorname{er}(1-e)=0$ since $\sigma_{i}$ is injective, so $e r=e r e$. Using a similar reasoning we can show that $r e=e r e$, and so $e r=r e$. Then, there exists $e^{2}=e \in R$ such that $r_{R}\left(\left\{a_{0}\right\}\right) \cap r_{R}\left(\left\{a_{1}\right\}\right) \cap \cdots \cap r_{R}\left(\left\{a_{t}\right\}\right)=e R$. The aim is to show that $r_{A}(f)=e A$. Using the $(\Sigma, \Delta)$-compatibility of $R$, and the equality $a_{j} e=0$ for $0 \leq j \leq t$, the expression $f e=\left(a_{0}+a_{1} X_{1}+\cdots+\right.$ $\left.a_{t} X_{t}\right) e=a_{0} e+a_{1} X_{1} e+\cdots+a_{t} X_{t} e$ is equal to zero by Remark 2.7. whence $f e=0$. Hence $e A \subseteq r_{A}(f)$. Now, consider an element $g \in r_{A}(f)$ given by the expression $g=b_{0}+b_{1} Y_{1}+\cdots+b_{s} Y_{s}$. Then $f g=0$, and since $R$ satisfies the condition (SA1), we have $a_{j} b_{k}=0$ for $0 \leq j \leq t$ and $0 \leq k \leq s$, that is, $b_{k} \in r_{R}\left(\left\{a_{j}\right\}\right)$, so $b_{k} \in e R$ for every $k$, which shows that $b_{k}=e r_{k}$, and hence $g=e r_{0}+e r_{1} Y_{1}+\cdots+e r_{s} Y_{s} \in e A$, which proves that $r_{A}(f) \subseteq e A$. Therefore, $r_{A}(f)=e A$.

Corollary 4.8 ([35, Theorem 3.12]). If $A$ is a bijective skew PBW extension of a $\Sigma$-rigid $R$, then $R$ is a p.p.-ring if and only if $A$ is a p.p.-ring. 
Example 4.9 ([15, Example 3.17]). Let $B=\mathbb{Z}_{2}[y] /\left\langle y^{2}\right\rangle$, where $\left\langle y^{2}\right\rangle$ is a principal ideal generated by $y^{2}$. The only idempotents of $B$ are $0+y^{2}$ and $1+y^{2}$. Since $r_{R}\left(\left\{y+\left\langle y^{2}\right\rangle\right\}\right)=\left\langle y+\left\langle y^{2}\right\rangle\right\rangle$ cannot be generated by an idempotent, $B$ is not quasiBaer and so it is not Baer. Now, let $\alpha$ be the identity map on $B$, and define an $\alpha$-derivation $\delta$ on $B$ by $\delta\left(y+\left\langle y^{2}\right\rangle\right)=1+\left\langle y^{2}\right\rangle$. Then $B$ is not $\delta$-compatible. Nevertheless, $B[x ; \alpha, \delta]=B[x ; \delta] \cong M_{2}\left(\mathbb{Z}_{2}\left[y^{2}\right]\right) \cong M_{2}\left(\mathbb{Z}_{2}[t]\right)$, where $M_{2}\left(\mathbb{Z}_{2}[t]\right)$ is a Baer ring, and hence $B[x ; \delta]$ is a Baer ring. This example shows that there exists a non- $\delta$-compatible ring $B$ which is not Baer, but $B[x ; \delta]$ is a Baer ring.

Next, we see one example of a $\sigma$-compatible left p.q.-Baer ring which is not $\sigma$-rigid.

Example 4.10 ([16, Example 1.1]). Let $B_{1}$ be a left p.q.-Baer ring, $D$ a domain and $B=T_{m}\left(B_{1}\right) \oplus D[y]$, where $T_{m}\left(B_{1}\right)$ is the upper $m \times m$ triangular matrix ring over $B_{1}$. Let $\sigma: D[y] \rightarrow D[y]$ be a monomorphism which is not surjective. Then $B$ is left p.q.-Baer, and if $\sigma$ is defined by $\bar{\sigma}(A \oplus f(y))=A \oplus(f(y))$ for each $A \in T_{n}\left(B_{1}\right)$ and $f(y) \in D[y]$, then $\sigma$ is a non surjective monomorphism and $B$ is a $\bar{\sigma}$-compatible ring which is not $\bar{\sigma}$-rigid because $B$ is not reduced.

Definition 4.11. Let $A$ be a skew PBW extension of $R$. We say that $R$ satisfies the (SQA1) condition if whenever $f A g=0$ for $f=a_{0}+a_{1} X_{1}+\cdots+a_{m} X_{m}$ and $g=b_{0}+b_{1} Y_{1}+\cdots+b_{t} Y_{t}$ elements of $A$, then $a_{i} R b_{j}=0$, for every $i, j$.

Following [18, for a ring $B, r \operatorname{Ann}_{B}(\operatorname{id}(B))=\left\{r_{B}(U) \mid U\right.$ is an ideal of $\left.B\right\}$, and similarly, $l \operatorname{Ann}_{B}(\operatorname{id}(B))=\left\{l_{B}(U) \mid U\right.$ is an ideal of $\left.B\right\}$. With this notation, we have the following proposition about $(\Sigma, \Delta)$-compatible rings which satisfy (SQA1). This proposition generalizes [18, Proposition 3.4] and [16, Proposition 2.5].

Theorem 4.12. If $A$ is a skew $P B W$ extension of $a(\Sigma, \Delta)$-compatible ring $R$, then the following statements are equivalent:

(i) $R$ satisfies condition ( $S Q A 1)$;

(ii) $\psi: r \operatorname{Ann}_{R}(\operatorname{id}(B)) \rightarrow r \operatorname{Ann}_{A}(\operatorname{id}(A))$, given by $C \rightarrow C A$, is bijective;

(iii) $\varphi: l \operatorname{Ann}_{R}(\operatorname{id}(B)) \rightarrow l \operatorname{Ann}_{A}(\operatorname{id}(A))$, given by $D \rightarrow A D$, is bijective.

Proof. (i) $\Rightarrow$ (ii) Consider an element $C \in r \operatorname{Ann}_{R}(\mathrm{id}(R))$. There exists an ideal $I$ of $R$ such that $C=r_{R}(I)$. By Proposition 3.8, we have the equality $r_{A}(A I A)=C A$. Hence, the application $\psi$ is well defined. Now, if $B \in r \operatorname{Ann}_{A}(\operatorname{id}(A))$, then there exists an ideal $J$ of $A$ with $B=r_{A}(J)$. Consider $B_{1}$ and $J_{1}$ the set of coefficients of elements of $B$ and $J$, respectively. The aim is to prove that $r_{R}\left(J_{1} R\right)=B_{1} R$. Let $f=a_{0}+a_{1} X_{1}+\cdots+a_{m} X_{m} \in J$ and $g=b_{0}+b_{1} Y_{1}+\cdots+b_{t} Y_{t} \in B$. Then $f A g=0$. Using the condition (SQA1) on $R$, we have $a_{i} R b_{j}=0$ for $0 \leq i \leq m$ and $0 \leq j \leq t$. Hence, $\left(J_{1} R\right)\left(B_{1} R\right)=0$, so $B_{1} \subseteq r_{R}\left(J_{1} R\right)$. By the $(\Sigma, \Delta)$ compatibility of $R$, we obtain $r_{R}\left(J_{1} R\right) \subseteq B_{1} R$, which shows that $r_{R}\left(J_{1} R\right)=B_{1} R$. Thus, $r_{A}(J)=\left(B_{1} R\right) A$.

(ii) $\Rightarrow$ (i) Consider the elements $f=a_{0}+a_{1} X_{1}+\cdots+a_{m} X_{m}, g=b_{0}+b_{1} Y_{1}+$ $\cdots+b_{t} Y_{t}$ of $A$ such that $f A g=0$. Then $g \in r_{A}(A f A)=C A$, where $C$ is an ideal of $R$. In this way, $b_{j} \in C$ for every $j$, that is, $f R b_{j}=0$. Lemma 3.11 guarantees that $a_{i} R b_{j}=0$, for $0 \leq i \leq m$ and $0 \leq j \leq t$. 
The proof of the equivalence (i) $\Leftrightarrow$ (iii) is similar.

Definition 4.13 ([16, Definition 2.6]). A submodule $N$ of a left $B$-module $M$ is called a pure submodule if $L \otimes_{B} N \rightarrow L \otimes_{B} M$ is a monomorphism for every right $B$-module $L$. An ideal $I$ of $B$ is said to be right s-unital if, for each $a \in I$, there is an $x \in I$ such that $a x=a$. If an ideal $I$ of $B$ is right $s$-unital, then for any finite subset $F$ of $I$ there exists an element $e \in I$ such that $x e=x$ for all $x \in F$. For an ideal $I$, the following conditions are equivalent: (i) $I$ is pure as a left ideal of $B$; (ii) $R / I$ is flat as a left $B$-module; (iii) $I$ is right $s$-unital.

The next Theorem 4.14 extends [18, Theorem 3.9] and [16, Theorem 2.7].

Theorem 4.14. If $A$ is a bijective skew PBW extension of a $(\Sigma, \Delta)$-compatible ring $R$, then the following assertions are equivalent:

(i) $l(R a)$ is pure as a left ideal of $B$ for any element $a \in R$;

(ii) $l(A f)$ is pure as a left ideal of $A$ for any element $f \in A$. In this case $R$ satisfies condition (SQA1).

Proof. Let us start proving that $R$ satisfies (SQA1). Consider the expression $\left(a_{0}+\right.$ $\left.a_{1} X_{1}+\cdots+a_{m} X_{m}\right) A\left(b_{0}+b_{1} Y_{1}+\cdots+b_{t} Y_{t}\right)=0$ with $a_{i}, b_{j} \in R$. Then

$$
\left(a_{0}+a_{1} X_{1}+\cdots+a_{m} X_{m}\right) R\left(b_{0}+b_{1} Y_{1}+\cdots+b_{t} Y_{t}\right)=0 .
$$

The leading coefficient of this expression is given by $a_{m} \sigma^{\alpha_{m}}\left(R b_{t}\right) c_{\alpha_{m}, \beta_{t}}=0$. Using the bijectivity of $A, a_{m} \sigma^{\alpha_{m}}\left(R b_{t}\right)=0$, and by the $\Sigma$-compatibility of $R$, we obtain $a_{m} R b_{t}=0$, that is, $a_{m} \in l_{R}\left(R b_{t}\right)$. From Remark 2.7 and Proposition 3.8 we have the equalities $a_{m} X_{m} R b_{0}=a_{m} X_{m} R b_{1} Y_{1}=\cdots=a_{m} X_{m} R b_{t-1} Y_{t-1}=0$, so we obtain the expression

$$
\left(a_{0}+a_{1} X_{1}+\cdots+a_{m} X_{m}\right) R\left(b_{0}+b_{1} Y_{1}+\cdots+b_{t-1} Y_{t-1}\right)=0 .
$$

Since $l_{R}\left(R b_{t}\right)$ is right $s$-unital, there exists $e_{t} \in l_{R}\left(R b_{t}\right)$ with $a_{m} e_{t}=a_{m}$.

Replacing $R$ by $e_{t} R$ in 4.1,

$$
\left(a_{0}+a_{1} X_{1}+\cdots+a_{m} X_{m}\right) e_{t} R\left(b_{0}+b_{1} Y_{1}+\cdots+b_{t-1} Y_{t-1}\right)=0
$$

we obtain its leading coefficient given by $a_{m} \sigma^{\alpha_{m}}\left(e_{t} R b_{t-1}\right) c_{\alpha_{m}, \beta_{t-1}}=0$, and since $A$ is bijective, $a_{m} \sigma^{\alpha_{m}}\left(e_{t} R b_{t-1}\right)=0$. By the $\Sigma$-compatibility of $R$, we obtain $a_{m} e_{t} R b_{t-1}=0$, i.e., $a_{m} \in l_{R}\left(e_{t} R b_{t-1}\right)$. Again, from Remark 2.7 (ii) and Proposition 3.8 we have the equalities $a_{m} X_{m} e_{t} R b_{0}=a_{m} X_{m} b_{1}=\cdots=a_{m} X_{m} b_{t-1} Y_{t-1}=$ 0 , so (4.2) takes the form

$$
\left(a_{0}+a_{1} X_{1}+\cdots+a_{m} X_{m}\right) e_{t} R\left(b_{0}+b_{1} Y_{1}+\cdots+b_{t-2} Y_{t-2}\right)=0 .
$$

In this way, $a_{m} \in l_{R}\left(R b_{t}\right) \cap l_{R}\left(e_{t} R b_{t-1}\right)$, and since $a_{m} e_{t}=a_{m}$, it follows that $a_{m} \in l_{R}\left(R b_{t}\right) \cap l_{R}\left(R b_{t-1}\right)$. Now, by assumption $l_{R}\left(R b_{t}\right)$ is right $s$-unital, so there exists $f \in l_{R}\left(R b_{t-1}\right)$ such that $a_{m} f=a_{m}$. If $e_{t-1}:=e_{t} f$, then $a_{m} e_{t-1}=a_{m} e_{t} f=$ $a_{m} f=a_{m}$, so $e_{t-1} \in l_{R}\left(R b_{t}\right) \cap l_{R}\left(R b_{t-1}\right)$.

Now, replacing $R$ by $e_{t-1} R$ in 4.1 , and using the reasoning above, we obtain the expression

$$
\left(a_{0}+a_{1} X_{1}+\cdots+a_{m} X_{m}\right) e_{t} R\left(b_{0}+b_{1} Y_{1}+\cdots+b_{t-2} Y_{t-2}\right)=0,
$$


with its leading coefficient $a_{m} \sigma^{\alpha_{m}}\left(e_{t} R b_{t-2}\right) c_{\alpha_{m}, \beta_{t-2}}=0$, and since $A$ is bijective, we obtain $a_{m} \sigma^{\alpha_{m}}\left(e_{t} R b_{t-2}\right)=0$. By the $\Sigma$-compatibility of $R$, we have $a_{m} e_{t} R b_{t-2}=0$, that is, $a_{m} \in l_{R}\left(e_{t} R b_{t-2}\right)$, whence $a_{m} \in l_{R}\left(R b_{t}\right) \cap l_{R}\left(R b_{t-1}\right) \cap$ $l_{R}\left(R b_{t-2}\right)$. Continuing in this way, we obtain $a_{m} R_{j}$ for $j=0, \ldots, t$. Using the $(\Sigma, \Delta)$-compatibility of $R$, the original expression takes the form

$$
\left(a_{0}+a_{1} X_{1}+\cdots+a_{m} X_{m}\right) A\left(b_{0}+b_{1} Y_{1}+\cdots+b_{t-1} Y_{t-1}\right)=0 .
$$

Using induction on $m+t$, we conclude that $a_{i} R b_{j}$ for every $i, j$, that is, $R$ satisfies (SQA1). Let us prove the equivalence of assertions (i) and (ii).

(i) $\Rightarrow$ (ii) Consider the elements $f, g \in l(A f)$ given by $f=a_{0}+a_{1} X_{1}+\cdots+a_{m} X_{m}$ and $g=b_{0}+b_{1} Y_{1}+\cdots+b_{t} Y_{t}$. Then $b_{j} R a_{i}=0$ for every $i, j$. By assumption, $l\left(R a_{i}\right)$ is right $s$-unital, so there exists $e_{i} \in l\left(R a_{i}\right)$ with $b_{j}=b_{j} e_{i}$ for $i=0, \ldots, m$. Let $e:=e_{0} e_{1} \cdots e_{m}$. Then $b_{j}=b_{j} e$ for every $j$. By Proposition 3.8, we obtain $e \in A f$ and $g e=g$, that is, $l(A f)$ is right $s$-unital.

(ii) $\Rightarrow$ (i) Let $a \in R$. Since $R$ is $(\Sigma, \Delta)$-compatible, we have $l_{R}(R a) \subseteq l_{A}(A a)$. In this way, for any $b \in l_{R}(R a)$ there exists an element $f \in A$ with $b f=b$. If $a_{0}$ is the constant term of $f$, then $b a_{0}=b$ and using the $(\Sigma, \Delta)$-compatibility of $R$, $a_{0} \in l_{R}(R a)$. Therefore $l_{R}(R a)$ is right $s$-unital.

Since quasi-Baer (left p.q.-Baer) rings satisfy the hypothesis of Theorem 4.14 we have the following result.

Theorem 4.15. If $A$ is a bijective skew $P B W$ extension of a $(\Sigma, \Delta)$-compatible ring $R$, then $R$ is quasi-Baer (left p.q.-Baer) if and only if $A$ is quasi-Baer (left p.q.-Baer). In this case, $R$ satisfies condition (SQA1).

Corollary 4.16 ([16, Corollary 2.8]). If $B$ is a $(\sigma, \delta)$-compatible ring, then $B$ is quasi-Baer (left p.q.-Baer) if and only if $B[x ; \sigma, \delta]$ is quasi-Baer (left p.q.-Baer).

Examples 4.17. The following examples show the importance of the $(\Sigma, \Delta)$ compatibility condition on $R$ in Theorem 4.15

(i) ([13, Example 2.8]). If $B=\mathbb{F}[t]$ is the polynomial ring over a field $\mathbb{F}$ and $\sigma$ is the endomorphism given by $\sigma(f(t))=f(0)$, then $B[x ; \sigma]$ is not a quasiBaer ring. This example shows that there is an example of a quasi-Baer ring $B$ and an endomorphism $\sigma$ of $B$ such that $B[x ; \sigma]$ is not a quasi-Baer ring.

(ii) (4, Example 11]). Let $B=\mathbb{Z}_{2}[t] /\left\langle t^{2}\right\rangle$, with the derivation $\delta$ such that $\delta(\bar{t})=1$ where $\bar{t}=t+\left\langle t^{2}\right\rangle$ in $B$, and $\mathbb{Z}_{2}[t]$ is the polynomial ring over the field $\mathbb{Z}_{2}$ of two elements. Consider the Ore extension $B[x ; \delta]$. If we set $e_{11}=\bar{t} x, e_{12}=\bar{t}, e_{21}=\bar{t} x^{2}+x$, and $e_{22}=1+\bar{t} x$ in $B[x ; \delta]$, then they form a system of matrix units in $B[x ; \delta]$. Now the centralizer of these matrix units in $B[x ; \delta]$ is $\mathbb{Z}_{2}\left[x^{2}\right]$. Therefore $B[x ; \delta] \cong M_{2}\left(\mathbb{Z}_{2}\left[x^{2}\right]\right) \cong M_{2}\left(\mathbb{Z}_{2}\right)[y]$, where $M_{2}\left(\mathbb{Z}_{2}\right)[y]$ is the polynomial ring over $M_{2}\left(\mathbb{Z}_{2}\right)$. So the ring $B[x ; \delta]$ is a Baer ring, but $B$ is not quasi-Baer. This example shows that there is a ring $B$ and a derivation $\delta$ of $B$ such that $B[x ; \delta]$ is a Baer ring but $B$ is not quasi-Baer. 
(iii) ([15, Example 2.1]). Let $B_{1}$ be a non-reduced left p.p.-ring, $D$ a domain and $B=B_{1} \oplus D[y]$. If $\sigma: D[y] \rightarrow D[y]$ is a monomorphism which is not surjective, then (i) $B$ is a left p.p.-ring (note that $B_{1}$ and $D[y]$ are left p.p.), and (ii) if $\bar{\sigma}: B \rightarrow B$ is the endomorphism defined by $\bar{\sigma}(a \oplus f(y))=$ $a \oplus \sigma(f(y))$ for every $a \in B_{1}$ and $f(y) \in D[y]$, then $\bar{\sigma}$ is a monomorphism of $B$ which is not surjective and $B$ is $\bar{\sigma}$-compatible and not $\bar{\sigma}$-rigid.

Since $\Sigma$-rigid rings are $(\Sigma, \Delta)$-compatible, we have immediately the following results established in the literature.

Corollary 4.18 ([19, Theorem 14]; [35, Theorems 3.10 and 3.13]). Let $R$ be a $\Sigma$-rigid ring. Then $R$ is quasi-Baer (left p.q.-Baer) if and only if $A$ is quasi-Baer (left p.q.-Baer).

Proof. The assertions follow from Proposition 3.4 and Theorem 4.15.

Finally, we will characterize the $(\Sigma, \Delta)$-compatibility for the classical ring of quotients of a bijective skew PBW extension.

Let us recall the key facts about noncommutative localization. If $B$ is a ring and $S$ is a multiplicative subset of $B\left(1 \in S, 0 \notin S, s s^{\prime} \in S\right.$ for every $\left.s, s^{\prime} \in S\right)$, then the left ring of fractions of $B$ exists if and only if two conditions hold: (i) given $a \in B$ and $s \in S$ with $a s=0$, there exists $s^{\prime} \in S$ such that $s^{\prime} a=0$; (ii) given $a \in B$ and $s \in S$, there exist $s^{\prime} \in S$ and $a^{\prime} \in B$ with $s^{\prime} a=a^{\prime} s$ (left Ore condition). If these conditions hold, then the left ring of fractions of $B$ with respect to $S$ is denoted by $S^{-1} B$, and its elements are classes denoted using fractions. More exactly, $\frac{a}{s}:=\frac{b}{t}$ are equal if and only if there exist $c, d \in B$ such that $c a=d b, c s=d t \in S$; $\frac{a}{s}+\frac{b}{t}:=\frac{c a+d b}{u}$, where $u:=c s=d t \in S$, for some $c, d \in B ; \frac{a}{s} \frac{b}{t}:=\frac{c b}{u s}$, where $u a=c t$, for some $u \in S$ and $c \in B$. Similarly, one defines the right Ore condition and hence the right ring of fractions of $B$. The nonzero divisor elements of $B$ are called regular and the set of regular elements of $B$ is denoted by $S_{0}(B)$. Recall that if $B$ is both left and right Ore, then its classical left ring of quotients $Q_{c l}^{l}(B)$ and its classical right ring of quotients $Q_{c l}^{r}(B)$ coincide, and it is denoted by $Q(B)$. A key result about the classical ring of quotients of $B$ is the common denominator property: if $B$ is a ring, $S \subset B$ is a multiplicative subset and $S^{-1} B$ exists, then any finite set $\left\{q_{1}, \ldots, q_{n}\right\}$ of elements of $S^{-1} B$ posses a common denominator, i.e., there exist $r_{1}, \ldots, r_{n} \in B$ and $s \in S$ such that $q_{i}=\frac{r_{i}}{s}$ for every $i$ (see [21] for a detailed treatment of localization in noncommutative rings).

Proposition 4.19 ([25, Lemma 2.6]). Let $A$ be a bijective skew PBW extension of a ring $R$. If $S \subseteq S_{0}(R)$ is a multiplicative subset of $R$ with $\sigma_{i}(S)=S$ for every $i=1, \ldots, n$, then

(a) If $S^{-1} R$ exists, then $S^{-1} A$ exists and it is a bijective skew $P B W$ extension of $S^{-1} R$, denoted $S^{-1} A=\sigma\left(S^{-1} R\right)\left\langle x_{1}^{\prime}, \ldots, x_{n}^{\prime}\right\rangle$, where $x_{i}^{\prime}:=\frac{x_{i}}{1}$, and the system of constants of $S^{-1} R$ is given by $c_{i, j}^{\prime}=\frac{c_{i, j}}{1}, c_{i, \frac{r}{s}}^{\prime}:=\frac{\sigma_{i}(r)}{\sigma_{i}(s)}$, for $1 \leq i, j \leq n$. The automorphisms $\overline{\sigma_{i}}$ of $S^{-1} R$ and the $\overline{\sigma_{i}}$-derivations $\overline{\delta_{i}}$ 
$(1 \leq i \leq n)$, are defined by $\overline{\sigma_{i}}\left(\frac{a}{s}\right):=\frac{\sigma_{i}(a)}{\sigma_{i}(s)}$, and $\overline{\delta_{i}}\left(\frac{a}{s}\right):=-\frac{\delta_{i}(s)}{\sigma_{i}(s)} \frac{a}{s}+\frac{\delta_{i}(a)}{\sigma_{i}(s)}$. Let $\bar{\Sigma}:=\left\{\overline{\sigma_{1}}, \ldots, \overline{\sigma_{n}}\right\}$ and $\bar{\Delta}:=\left\{\overline{\delta_{1}}, \ldots, \overline{\delta_{n}}\right\}$.

(b) If $R S^{-1}$ exists, then $A S^{-1}$ exists and it is a bijective skew $P B W$ extension of $R S^{-1}$, denoted $A S^{-1}=\sigma\left(R S^{-1}\right)\left\langle x_{1}^{\prime \prime}, \ldots, x_{n}^{\prime \prime}\right\rangle$, where $x_{i}^{\prime \prime}:=\frac{x_{i}}{1}$, and the system of constants of $S^{-1} R$ is given by $c_{i, j}^{\prime \prime}=\frac{c_{i, j}}{1}, c_{i, \frac{r}{s}}^{\prime \prime}:=\frac{\sigma_{i}(r)}{\sigma_{i}(s)}$, for $1 \leq i, j \leq n$. The automorphisms $\overline{\sigma_{i}}$ of $S^{-1} R$ and the $\overline{\sigma_{i}}$-derivations $\overline{\delta_{i}}$ $(1 \leq i \leq n)$, are defined by $\overline{\sigma_{i}}\left(\frac{a}{s}\right):=\frac{\sigma_{i}(a)}{\sigma_{i}(s)}$, and $\overline{\delta_{i}}\left(\frac{a}{s}\right):=-\frac{\sigma_{i}(a)}{\sigma_{i}(s)} \frac{\delta_{i}(s)}{s}+\frac{\delta_{i}(a)}{s}$. Let $\bar{\Sigma}:=\left\{\overline{\sigma_{1}}, \ldots, \overline{\sigma_{n}}\right\}$ and $\bar{\Delta}:=\left\{\overline{\delta_{1}}, \ldots, \overline{\delta_{n}}\right\}$.

If no confusion arises, we simply denote $x_{i}^{\prime}$ and $x_{i}^{\prime \prime}$ by $x_{i}$ for $1 \leq i \leq n$. Now, analogously to the definition of $(\Sigma, \Delta)$-compatibility, we consider the notion of $(\bar{\Sigma}, \bar{\Delta})$-compatibility for the classical quotient ring $Q(R)$ of $R$.

Theorem 4.20. Let $A$ be a bijective skew $P B W$ extension of a ring $R$. Suppose that the classical ring of quotients $Q(R)$ of $R$ exists. If $R$ is $(\Sigma, \Delta)$-compatible, then the classical quotient ring $Q(R)$ of $R$ is $(\bar{\Sigma}, \bar{\Delta})$-compatible.

Proof. If $R$ is $(\Sigma, \Delta)$-compatible, and $a c^{-1} b d^{-1}=0$, then we obtain $a c^{-1} b=$ 0 . Note that we also have $a c^{-1}=c^{-1} a^{\prime}$, so $c^{-1} a^{\prime} b=0$, whence $a^{\prime} b=0$. By assumption, $R$ is $\Sigma$-compatible, so $a^{\prime} \sigma^{\alpha}(b)=0$, for every $\alpha \in \mathbb{N}^{n}$. Hence, $c^{\prime-1} a^{\prime} \sigma^{\alpha}(b)\left(\sigma^{\alpha}(d)\right)^{-1}=0$, i.e., $a c^{-1} \sigma^{\alpha}(b)\left(\sigma^{\alpha}(d)\right)^{-1}=0$, that is, $a c^{-1} \overline{\sigma^{\alpha}}\left(b d^{-1}\right)=$ 0 . In a similar way, we can see that $a c^{-1} \overline{\sigma^{\alpha}}\left(b d^{-1}\right)=0$ implies that $a c^{-1} b d^{-1}=0$, which means that $Q(R)$ is $\bar{\Sigma}$-compatible.

Now, if $a c^{-1} b d^{-1}=0$, then $a c^{-1} \overline{\delta^{\beta}}\left(b d^{-1}\right)=a c^{-1}\left(\delta^{\beta}(b)-b \delta^{\beta}(d) d^{-1}\right)\left(\sigma^{\beta}(d)\right)^{-1}$. Using a similar reasoning as above, $a^{\prime} b=0$, where $a c^{-1}=c^{\prime-1} a^{\prime}$, and hence $a^{\prime} \delta^{\beta}(b)=0$. Therefore $c^{-1} a^{\prime} \delta^{\beta}(b)\left(\sigma^{\beta}(d)\right)^{-1}=0$, which implies that $a c^{-1} \delta^{\beta}(b)\left(\sigma^{\beta}(d)\right)^{-1}=0$. Note that $a^{\prime} b=0$ implies that $a^{\prime} b \delta^{\beta}(d) d^{-1}\left(\sigma^{\beta}(d)\right)^{-1}=0$, and so $a c^{-1} b \delta^{\beta}(d) d^{-1}\left(\sigma^{\beta}(d)\right)^{-1}=0$. In this way, $a c^{-1} \overline{\delta^{\beta}}\left(b d^{-1}\right)=a c^{-1}\left(\delta^{\beta}(b)-\right.$ $\left.b \delta^{\beta}(d) d^{-1}\right)\left(\sigma^{\beta}(d)\right)^{-1}=0$, that is, $Q(R)$ is $\bar{\Delta}$-compatible. This fact concludes the proof.

\section{ExAmples}

In this section we present some remarkable examples of skew PBW extensions which cannot be expressed as Ore extensions (see [27] for a detailed reference of every example, and a more complete list of noncommutative rings).

(a) Let $k$ be a commutative ring and $\mathfrak{g}$ a finite dimensional Lie algebra over $k$ with basis $\left\{x_{1}, \ldots, x_{n}\right\}$. The universal enveloping algebra of $\mathfrak{g}$, denoted $\mathcal{U}(\mathfrak{g})$, is a skew PBW extension of $k$, since $x_{i} r-r x_{i}=0, x_{i} x_{j}-x_{j} x_{i}=$ $\left[x_{i}, x_{j}\right] \in \mathfrak{g}=k+k x_{1}+\cdots+k x_{n}, r \in k$, for $1 \leq i, j \leq n$. In particular, the universal enveloping algebra of a Kac-Moody Lie algebra is a skew PBW extension of a polynomial ring.

(b) The universal enveloping $\operatorname{ring} \mathcal{U}(V, R, \mathbb{k})$, where $R$ is a $\mathbb{k}$-algebra and $V$ is a $\mathbb{k}$-vector space which is also a Lie ring containing $R$ and $\mathbb{k}$ as Lie ideals with suitable relations. The enveloping $\operatorname{ring} \mathcal{U}(V, R, \mathbb{k})$ is a finite skew PBW extension of $R$ if $\operatorname{dim}_{\mathbb{k}}(V / R)$ is finite. 
(c) Let $k, \mathfrak{g},\left\{x_{1}, \ldots, x_{n}\right\}$ and $\mathcal{U}(\mathfrak{g})$ be as in the previous example; let $R$ be a $k$-algebra containing $k$. The tensor product $A:=R \otimes_{k} \mathcal{U}(\mathfrak{g})$ is a skew PBW extension of $R$, and it is a particular case of crossed product $R * \mathcal{U}(\mathfrak{g})$ of $R$ by $\mathcal{U}(\mathfrak{g})$, which is a skew PBW extension of $R$.

(d) The twisted or smash product differential operator ring $R \#_{\sigma} \mathcal{U}(\mathfrak{g})$, where $\mathfrak{g}$ is a finite-dimensional Lie algebra acting on $R$ by derivations, and $\sigma$ is a Lie 2-cocycle with values in $R$.

(e) Diffusion algebras arise in physics as a possible way to understand a large class of 1-dimensional stochastic process. A diffusion algebra $\mathcal{A}$ with parameters $a_{i j} \in \mathbb{C} \backslash\{0\}, 1 \leq i, j \leq n$, is an algebra over $\mathbb{C}$ generated by variables $x_{1}, \ldots, x_{n}$ subject to relations

$$
a_{i j} x_{i} x_{j}-b_{i j} x_{j} x_{i}=r_{j} x_{i}-r_{i} x_{j},
$$

whenever $i<j, b_{i j}, r_{i} \in \mathbb{C}$ for all $i<j$. $\mathcal{A}$ admits a PBW-basis of standard monomials $x_{1}^{i_{1}} \cdots x_{n}^{i_{n}}$, that is, $\mathcal{A}$ is a diffusion algebra if these standard monomials are a $\mathbb{C}$-vector space basis for $\mathcal{A}$. From Definition 2.1. (iii) and (iv), it is clear that the family of skew PBW extensions are more general than diffusion algebras. We will denote $q_{i j}:=\frac{b_{i j}}{a_{i j}}$. The parameter $q_{i j}$ can be a root of unity if and only if it is equal to 1 . It is therefore reasonable to assume these parameters not to be a root of unity other than 1 . If all the coefficients $q_{i j}$ are nonzero, then the corresponding diffusion algebra has a PBW basis of standard monomials $x_{1}^{i_{1}} \cdots x_{n}^{i_{n}}$, and hence these algebras are skew PBW extensions. More precisely, $\mathcal{A} \cong \sigma(\mathbb{C})\left\langle x_{1}, \ldots, x_{n}\right\rangle$.

\section{REFERENCES}

[1] Anderson, D. D., Camillo, V. Armendariz rings and Gaussian rings, Comm. Algebra 26 (1998), no. 7, 2265-2272. MR 1626606

[2] Annin, S. Associated primes over skew polynomial rings, Comm. Algebra 30 (2002), no. 5, 2511-2528. MR 1904650

[3] Armendariz, E. P. A note on extensions of Baer and P.P.-rings, J. Austral. Math. Soc. 18 (1974), 470-473. MR 0366979.

[4] Armendariz, E. P., Koo, H. K., Park, J. K. Isomorphic Ore extensions, Comm. Algebra 15 (1987), no. 12, 2633-2652. MR 0917758

[5] Birkenmeier, G. F. Baer rings and quasicontinuous rings have a MDSN, Pacific J. Math. 97 (1981), no. 2, 283-292. MR 0641158

[6] Birkenmeier, G. F., Kim, J. Y., Park, J. K. On polynomial extensions of principally quasiBaer rings, Kyungpook Math. J. 40 (2000), no. 2, 247-253. MR 1803098

[7] Birkenmeier, G. F., Kim, J. Y., Park, J. K. On quasi-Baer rings. In: Algebra and its applications (Athens, OH, 1999), 67-92, Contemp. Math., 259, Amer. Math. Soc., Providence, RI, 2000. MR 1778495

[8] Birkenmeier, G. F., Kim, J. Y., Park, J. K. Principally quasi-Baer rings, Comm. Algebra 29 (2001), no. 2, 639-660. MR 1841988

[9] Birkenmeier, G. F., Kim, J. Y., Park, J. K. Polynomial extensions of Baer and quasi-Baer rings, J. Pure Appl. Algebra 159 (2001), no. 1, 25-42. MR 1823504

[10] Clark, W. E. Twisted matrix units semigroup algebras, Duke Math. J. 34 (1967), 417-423. MR 0214626 
[11] Curado, E. M. F., Hassouni, Y., Rego-Monteiro, M. A., Rodrigues, L. M. C. S. Generalized Heisenberg algebra and algebraic method: The example of an infinite square-well potential, Phys. Lett. A 372 (2008), no. 19, 3350-3355. MR 2414703

[12] Gallego, C., Lezama, O. Gröbner bases for ideals of $\sigma$-PBW extensions, Comm. Algebra 39 (2011), no. 1, 50-75. MR 2770878.

[13] Han, J., Hirano, Y., Kim, H. Semiprime Ore extensions, Comm. Algebra 28 (2000), no. 8, 3795-3801. MR 1767590.

[14] Han, J., Hirano, Y., Kim, H., Some results on skew polynomial rings over a reduced ring. In: International Symposium on Ring Theory (Kyongju, 1999), 123-129, Trends Math., Birkhäuser Boston, Boston, MA, 2001. MR 1851197.

[15] Hashemi, E., Moussavi, A., Haj Seyyed Javadi, H. Polynomial Ore extensions of Baer and p.p.-rings, Bull. Iranian Math. Soc. 29 (2003), no. 2, 65-86. MR 2141851

[16] Hashemi, E., Moussavi, A. Polynomial extensions of quasi-Baer rings, Acta Math. Hungar. 107 (2005), no. 3, 207-224. MR 2148584.

[17] Hirano, Y. On ordered monoid rings over a quasi-Baer ring, Comm. Algebra 29 (2001), no. 5, 2089-2095. MR 1837964.

[18] Hirano, Y. On annihilator ideals of a polynomial ring over a noncommutative ring, J. Pure Appl. Algebra 168 (2002), no. 1, 45-52. MR 1879930

[19] Hong, C. Y., Kim, N. K., Kwak, T. K. Ore extensions of Baer and p.p.-rings, J. Pure Appl. Algebra 151 (2000), no. 3, 215-226. MR 1776431

[20] Hong, C. Y., Kim, N. K., Kwak, T. K. On skew Armendariz rings, Comm. Algebra 31 (2003), no. 1, 103-122. MR 1969216

[21] Jategaonkar, A. V. Localization in Noetherian rings, London Mathematical Society Lecture Note Series, 98, Cambridge University Press, Cambridge, 1986. MR 0839644.

[22] Kaplansky, I. Rings of Operators. W. A. Benjamin, New York-Amsterdam, 1968. MR 0244778

[23] Kim, N. K., Lee, Y. Armendariz rings and reduced rings, J. Algebra 223 (2000), no. 2, 477-488. MR 1735157 .

[24] Krempa, J. Some examples of reduced rings, Algebra Colloq. 3 (1996), no. 4, 289-300. MR 1422968

[25] Lezama, O., Acosta, J. P., Chaparro, C., Ojeda, I., Venegas, C. Ore and Goldie theorems for skew PBW extensions, Asian-Eur. J. Math. 6 (2013), no. 4, 1350061, 20 pp. MR 3149278.

[26] Lezama, O., Acosta, J. P., Reyes, A. Prime ideals of skew PBW extensions, Rev. Un. Mat. Argentina 56 (2015), no. 2, 39-55. MR 3431813

[27] Lezama, O., Reyes, A. Some homological properties of skew PBW extensions, Comm. Algebra 42 (2014), no. 3, 1200-1230. MR 3169625

[28] Matczuk, J. A characterization of $\sigma$-rigid rings, Comm. Algebra 32 (2004), no. 11, 4333-4336. MR 2102452

[29] Niño, D., Reyes, A. (2017). Some ring theoretical properties of skew Poincaré-Birkhoff-Witt extensions, Bol. Mat. (N.S.), to appear.

[30] Rege, M. B., Chhawchharia, S. Armendariz rings, Proc. Japan Acad. Ser. A Math. Sci. 73 (1997), no. 1, 14-17. MR 1442245.

[31] Reyes, A., Ring and Module Theoretical Properties of $\sigma-P B W$ Extensions, PhD Thesis, Universidad Nacional de Colombia, Bogotá, 2013.

[32] Reyes, A. Gelfand-Kirillov dimension of skew PBW extensions, Rev. Colombiana Mat. 47 (2013), no. 1, 95-111. MR 3078702

[33] Reyes, A. Uniform dimension over skew PBW extensions, Rev. Colombiana Mat. 48 (2014), no. 1, 79-96. MR 3230798.

[34] Reyes, A. Jacobson's conjecture and skew PBW extensions, Rev. Integr. Temas Mat. 32 (2014), no. 2, 139-152. MR 3288858.

[35] Reyes, A. Skew PBW extensions of Baer, quasi-Baer, p.p. and p.q.-rings, Rev. Integr. Temas Mat. 33 (2015), no. 2, 173-189. MR 3445965 
[36] Reyes, A., Suárez, H. A note on zip and reversible skew PBW extensions, Bol. Mat. (N.S.) 23 (2016), no. 1, 71-79. MR 3547275

[37] Reyes, A., Suárez, H. Some remarks about the cyclic homology of skew PBW extensions, Ciencia en Desarrollo 7 (2016), no. 2, 99-107.

[38] Reyes, A., Suárez, H. Armendariz property for skew PBW extensions and their classical ring of quotients, Rev. Integr. Temas Mat. 34 (2016), no. 2, 147-168. MR 3621421.

[39] Reyes, A., Suárez, H. PBW bases for some 3-dimensional skew polynomial algebras, Far East J. Math. Sci. 101 (2017), no. 6, 1207-1228.

[40] Reyes, A., Suárez, H. Bases for quantum algebras and skew Poincaré-Birkhoff-Witt extensions, Momento no. 54 (2017), 54-75.

[41] Reyes, A., Suárez, H. $\sigma$-PBW Extensions of skew Armendariz rings, Adv. Appl. Clifford Algebr. (2017). https://doi.org/10.1007/s00006-017-0800-4

[42] Reyes, A., Suárez, H. Enveloping algebra and skew Calabi-Yau algebras over skew PoincaréBirkhoff-Witt extensions, Far East J. Math. Sci. 102 (2017), no. 2, 373-397.

[43] Suárez, H., Lezama, O., Reyes, A. Some relations between N-Koszul, Artin-Schelter regular and Calabi-Yau algebras with skew PBW extensions, Ciencia en Desarrollo 6 (2015), no. 2, 205-213.

[44] Suárez, H., Reyes, A. A generalized Koszul property for skew PBW extensions, Far East J. Math. Sci. 101 (2017), no. 2, 301-320.

[45] Suárez, H., Reyes, A. Koszulity for skew PBW extensions over fields, JP J. Algebra Number Theory Appl. 39 (2017), no. 2, 181-203.

[46] Yakoub, L'moufadal B., Louzari, M., On quasi-Baer rings of Ore extensions, East-West J. Math. 8 (2006), no. 2, 119-127. MR 2442419.

\author{
A. Reyes $\bowtie$ \\ Department of Mathematics, Universidad Nacional de Colombia \\ mareyesv@unal. edu.co
}

H. Suárez

School of Mathematics and Statistics, Universidad Pedagógica y Tecnológica de Colombia

hector.suarez@uptc.edu.co

Received: October 12, 2016

Accepted: September 15, 2017 